\title{
Coupling dependence of jet quenching in hot strongly-coupled gauge theories
}

\author{
Peter Arnold, Phillip Szepietowski, and Diana Vaman \\ Department of Physics, University of Virginia, \\ Box 400714, Charlottesville, Virginia 22904, USA
}

(Dated: October 15, 2018)

\begin{abstract}
Previous top-down studies of jet stopping in strongly-coupled QCD-like plasmas with gravity duals have been in the infinite 't Hooft coupling limit $\lambda \rightarrow \infty$. They have found that, though a wide range of jet stopping distances are possible depending on initial conditions, the maximum jet stopping distance $\ell_{\max }$ scales with energy as $E^{1 / 3}$ at large energy. But it has always been unclear whether the large-coupling and high-energy limits commute. In this paper, we use the string $\alpha^{\prime}$ expansion in AdS-CFT to study the corrections to the $\lambda=\infty$ result in powers of $1 / \lambda$. For the particular type of "jets" that we study, we find that (i) the naive expansion in $1 / \lambda$ breaks down for certain initial conditions but (ii) the relative corrections to the maximum stopping distance are small when $1 / \lambda$ is small. More specifically, we find that the expansion in $1 / \lambda$ is well behaved for jets whose stopping distance $\ell_{\text {stop }}$ is in the range $\lambda^{-1 / 6} \ell_{\max } \ll \ell_{\text {stop }} \lesssim \ell_{\text {max }}$, but the expansion breaks down (and the fate of $\lambda=\infty$ results is uncertain) for jets created in such a way that $\ell_{\text {stop }} \ll$ $\lambda^{-1 / 6} \ell_{\max }$. The analysis requires assessing the effects of all higher-derivative corrections to the supergravity action for the gravity dual.
\end{abstract}




\section{INTRODUCTION AND RESULTS}

An important theoretical question for the study of jet quenching in quark-gluon plasmas is how far a high-momentum low-mass excitation (such as a high-energy massless parton with $E \gg T$ ) can propagate through the plasma before losing its energy to the plasma and thermalizing. A variety of authors $[1-6]^{1}$ have used gauge-gravity duality to study this problem in the strong coupling limit $\lambda \equiv N_{\mathrm{c}} g_{\mathrm{YM}}^{2} \rightarrow \infty$ of large- $N_{\mathrm{c}}, \mathcal{N}=4$ supersymmetric Yang-Mills (SYM) and related QCD-like plasmas. The exact stopping distance depends on the exact nature of the excitation, but it has been found that the maximum stopping distance scales with energy as $E^{1 / 3}$ (in contrast to the weak-coupling result, which is $E^{1 / 2}$ up to $\operatorname{logarithms^{2}}$ ). It is an interesting and instructive result that the power of energy depends on coupling, but there is a potential loophole to this conclusion, which has to do with orders of limits. The work on this problem to date has implicitly taken the $\lambda \rightarrow \infty$ and $N_{\mathrm{c}} \rightarrow \infty$ limits first, and only then considered the limit $E / T \rightarrow \infty$ of high-energy excitations. Consider two possibilities for large but finite $\lambda$ : (i) the maximum stopping distance grows like $E^{1 / 3}$ for arbitrarily large energies, versus (ii) it first grows like $E^{1 / 3}$ for $T \ll E \ll \lambda^{n} T$ (for some $n>0$ ) but then behaves differently for $E \gg \lambda^{n} T$. We cannot distinguish between these possibilities with only $\lambda=\infty$ results. Yet the distinction is an important one if there is any qualitative application of such results to real QCD plasmas, since $\lambda$ in the real world is not a fantastically large number. Similar issues arises with $N_{\mathrm{c}}$ : Might jet stopping be qualitatively different for $E \gg N_{\mathrm{c}}^{n} T$ ?

In this paper, we will keep ${ }^{3} N_{\mathrm{c}}=\infty$ and address the question of what happens when $\lambda$ is large but not infinite. In the gravity dual, this will require considering the effect of string corrections to the supergravity action. These corrections correspond to higherderivative terms in the supergravity action, such as the 4th power of the Riemann curvature. Formally, the effects of higher and higher derivative corrections to the supergravity action are suppressed by more and more factors of $1 / \sqrt{\lambda}$, but these suppressions might be compensated by large factors of $E / T$ in the jet stopping problem.

For the particular type of "jet" excitations that we will study, fig. 1 summarizes our results. This figure depicts the parametric importance of corrections as a function of the $\lambda=\infty$ result $\ell_{\text {stop }}$ for the stopping length of the jet. Parametrically, the maximum scale for $\ell_{\text {stop }}$ is

$$
\ell_{\max } \sim \frac{E^{1 / 3}}{T^{4 / 3}}
$$

The straight lines on this log-log plot represent simple power-law dependencies on the stop-

1 See also Sin and Zahed 7] for the earliest attempt we are aware of to discuss jet stopping in the context of gauge-gravity duality. See also ref. [8]. In our paper, we will only consider analogs of light-particle jets and will not study the heavy-particle case. For $\lambda=\infty$ analysis of the latter, see, for example, refs. [9, 10] and references therein.

2 A specific calculation for non-supersymmetric QCD of the stopping distance at weak coupling in the high-energy limit may be found in ref. [11]. However, the scaling of this result was implicit in the early pioneering work of refs. [12, 13] on bremsstrahlung and energy loss rates in QCD plasmas. The introduction of supersymmetry will not change the conclusion that the stopping distance scales as $E^{1 / 2}$ (up to logarithms) at weak coupling.

3 For a discussion of one potential source of $1 / N_{\mathrm{c}}$ corrections to jet propagation, see Shuryak, Yee, and Zahed [18]. 


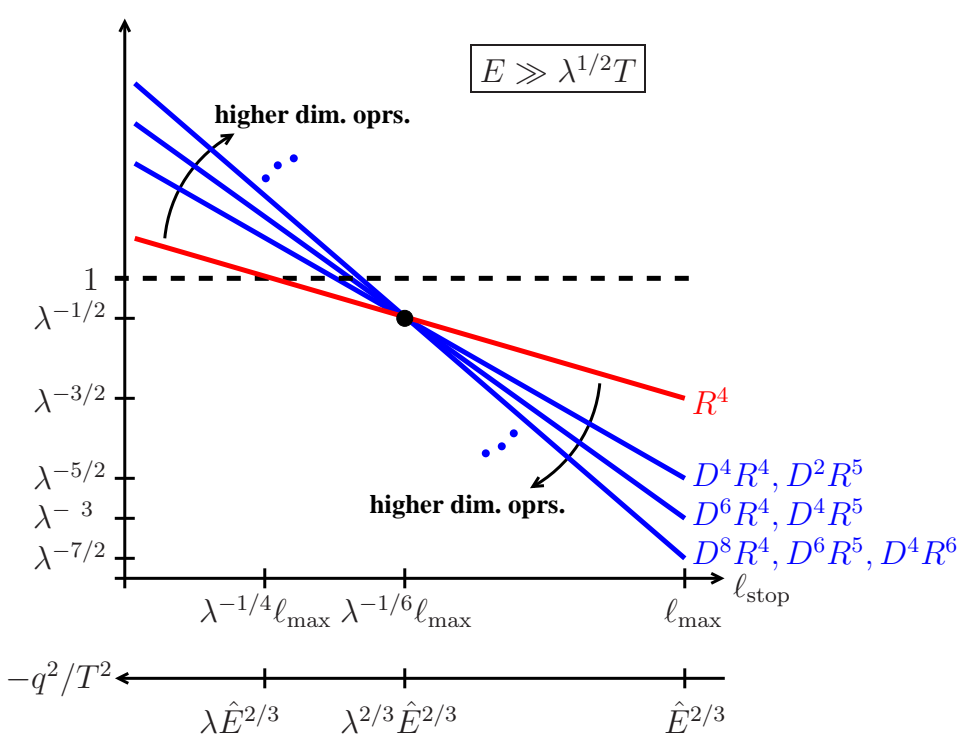

FIG. 1: A parametric picture of the relative importance of higher-derivative corrections to the low-energy supergravity action as a function of the stopping distance $\ell_{\text {stop }}$ (using the $\lambda=\infty$ result for $\left.\ell_{\text {stop }}\right)$. The axis are both logarithmic, and an importance of 1 indicates that the individual correction would, by itself, significantly modify the $\lambda=\infty$ analysis. Our measure of "importance" is explained in section IIC. Also shown, as an alternative horizontal axis, is the 4-dimensional virtuality $-q^{2}$ of the source that created the jet, where $\hat{E} \equiv E / T$.

ping distance. The first correction to the 10-dimensional low-energy supergravity action for the gravity dual to $\mathcal{N}=4$ super-Yang-Mills is of the form $R^{4}$ [22] (plus other terms related by supersymmetry), where $R^{4}$ is short-hand for a particular combination of contractions of four powers of the Riemann tensor. We've labeled each curve in fig. 1 with a few examples of the type of higher-derivative correction that contributes to each. (As we will see, the importance to jet stopping of a correction term in the supergravity action is determined by more than just its dimension, and we've only shown examples from among the most important terms of each dimension.) We will explain later exactly what we mean by the importance of an operator, denoted by the vertical axis. It is not quite the same thing as the relative change in stopping distance due to that operator, but, when the "importance" is small, the effect on the stopping distance will also be small. Finally, we note that fig. 1 assumes the high-energy limit $E \gg \lambda^{1 / 2} T$. If $E \ll \lambda^{1 / 2} T$ (which is equivalent to $\lambda^{-1 / 6} \ell_{\max } \ll 1 / T$ ), then the corrections to $\lambda=\infty$ jet stopping results remain small from $\ell_{\text {stop }} \sim \ell_{\text {max }}$ all the way down to $\ell_{\text {stop }} \sim 1 / T$, which is the smallest jet stopping distance that we will consider. ${ }^{4}$

From fig. 1, we conclude that corrections to the usual $\lambda=\infty$ analysis of jet stopping are small for $\ell_{\text {stop }} \gg \lambda^{-1 / 6} \ell_{\max }$. This includes in particular the case of jets that travel the maximum stopping distance, $\ell_{\text {stop }} \sim \ell_{\max }$, given by (1.1). For jets with smaller stopping

4 At a technical level, we define where the jet stops [4, 5] following Chesler et al. [3, 19] as the location where the jet's energy and momentum and charge first begin to evolve hydrodynamically. Since hydrodynamics is an effective theory only on distance scales $\gg 1 / T$ at strong coupling, it does not make sense to apply this definition to stopping distances small compared to $1 / T$. 


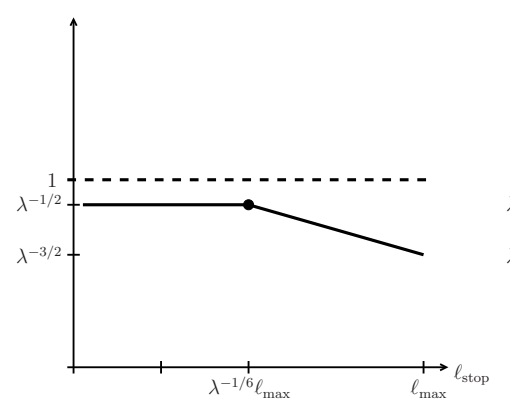

(a)

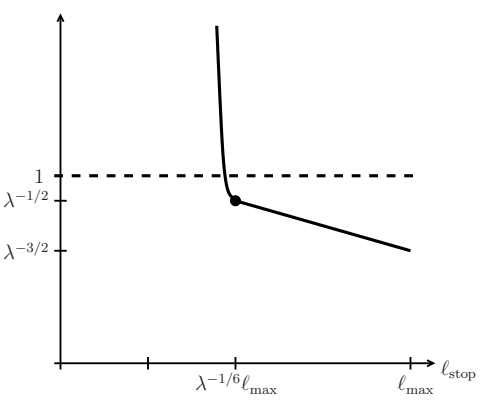

(b)

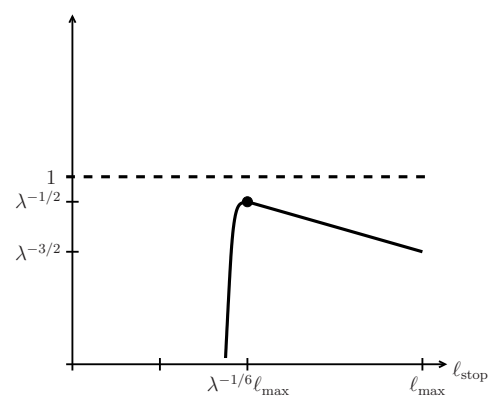

(c)

FIG. 2: Like fig. 1 but showing some different behaviors that the total correction (summing all higher-derivative corrections) might conceivably have.

distances, $\ell_{\text {stop }} \ll \lambda^{-1 / 6} \ell_{\max }$, the conclusion is uncertain. As one decreases $\ell_{\text {stop }}$ down from $\ell_{\max }$, all the corrections become the same order at $\ell_{\text {stop }} \sim \lambda^{-1 / 6} \ell_{\max }$, but the corrections are individually all small (order $\lambda^{-1 / 2}$ ) at this scale. At lower $\ell_{\text {stop }}$, the expansion in higherderivative corrections is no longer useful, and one can imagine different behaviors depending on how that expansion sums. Perhaps the relative size of the total correction to the $\lambda=\infty$ result flattens out, as depicted in fig. 2 a. Perhaps the corrections sum to give rapid (e.g. exponential) growth, as in fig. 2b. Perhaps they sum to give rapid suppression, as in fig. 2 r. Figuring out what happens for $\ell_{\text {stop }} \ll \lambda^{-1 / 6} \ell_{\max }$ would presumably involve a full stringtheory analysis of the problem, which is beyond the scope of this paper. Here we just note that the validity of the $\lambda=\infty$ results for $\ell_{\text {stop }} \ll \lambda^{-1 / 6} \ell_{\max }$ is unclear: it might be that $\lambda=\infty$ remains a good approximation in that case, as in figs. 2 a and c, or fails completely, as in fig. $2 \mathrm{~b}$.

We should emphasize at the outset that finding the parametric dependence of corrections shown in fig. 1 will not depend on knowing details of the precise form of higher-derivative corrections to the supergravity action, nor on details of their precise effects on the $\mathrm{AdS}_{5^{-}}$ Schwarzschild background. Such details are not known for corrections involving high powers of curvature. And though we have taken care in fig. 1 to only depict higher-derivative corrections that actually appear as string corrections to Type IIB supergravity, ${ }^{5}$ which is the case relevant to $\mathcal{N}=4 \mathrm{SYM}$, our qualitative results do not depend on these details either. Our results will follow from general arguments concerning any expansion of the supergravity action in higher-derivative corrections.

Our results in fig. 1 are parametric in nature. In fact, given the types of "jets" that we will study, the maximum stopping distance scale $\ell_{\max }$ given by (1.1) will only be defined parametrically. It is the distance scale beyond which the amount of charge that a highlypenetrating jet deposits in the medium, on average, begins to fall exponentially. One may define a related scale $\ell_{\text {tail }}$ by characterizing this exponential fall-off as

$$
\operatorname{deposition}\left(x^{3}\right) \sim \text { prefactor } \times e^{-x^{3} / \ell_{\text {tail }}} \quad \text { for } \quad x^{3} \gg \ell_{\max }
$$

5 For a nice summary of higher-dimensional gravitational corrections in Type II supergravity generated by tree-level string amplitudes (i.e. in the $N_{\mathrm{c}}=\infty$ limit), see Table 1 of Stieberger [20]. Though not relevant to the $N_{\mathrm{c}}=\infty$ case we are discussing, a nice discussion of corrections generated from one-loop string amplitudes may be found in Richards [21]. 
for a jet moving in the $x^{3}$ direction. Fig. 1 indicates that the expansion in higher-derivative corrections should be well-behaved around $\ell_{\max }$, and correspondingly the corrections to $\ell_{\text {tail }}$ should be well-behaved. We will explicitly compute the leading, $R^{4}$ correction to $\ell_{\text {tail }}$. The precise result depends on details of the type of source used to initially create the jets. As an example, here is the result that we will find if we imagine (as a thought experiment in the field theory) creating a jet in the $\mathcal{N}=4$ SYM plasma via the decay of a high momentum, slightly off-shell graviton:

$$
\ell_{\text {tail }}=\ell_{\text {tail }}^{\lambda=\infty}\left[1+47.162 \lambda^{-3 / 2}+O\left(\lambda^{-5 / 2}\right)\right] .
$$

This result for $\ell_{\text {tail }}$ increases with decreasing $\lambda$.

The first part of this paper will be devoted to a general analysis of all higher-derivative corrections, as shown in fig. 1. For that analysis, it will be easiest to focus on the case $\ell_{\text {stop }} \ll \ell_{\text {max }}$, where it turns out that one may simplify the analysis by using a geometric optics approximation. (Parametric results for $\ell_{\text {stop }} \sim \ell_{\max }$ may then be obtained by extrapolation.) In section II, we review our basic framework [4] for creating high-momentum excitations ("jets") and the simplest version [5] of the corresponding calculation of $\lambda=\infty$ stopping distances. (Similarities and differences with the methods of other authors will be briefly summarized.) We will then be in a position to explain the nature of the "importance" measure sketched in fig. 1, as well as some assumptions that we make in our analysis. We also discuss the source we use in the field theory problem to create our jets, which we choose in a way that simplifies the discussion of the effects of higher-derivative corrections in the gravity dual. In section III, we start by discussing the first higher-derivative correction to the supergravity theory, which is an $R^{4}$ term in the gravity action. We show how this correction generates the corresponding (red) curve in fig. 1. Section IV] then extends the analysis to higher derivative terms of the form $D^{2 n} R^{4}$ in the gravity action. Section $\mathrm{V}$ moves on to higher powers of the curvature, $D^{2 n} R^{m}$. In section $\mathrm{VI}$, we briefly present another way of looking at when the expansion in supergravity corrections breaks down.

The second part of this paper presents the calculation of the scale $\ell_{\text {tail }}(1.2)$ of exponential tails. Section VII begins by explaining more carefully the definition of $\ell_{\text {tail }}$ and the situations that produce the desired behavior (1.2). Precise extraction of the fall-off (1.2) is beyond the range of validity of the geometric optics approximation, but one may instead extract results by solving for quasi-normal mode solutions to the appropriate linearized supergravity equations of motion. We carry out this calculation for the leading correction to $\ell_{\text {tail }}$ in powers of $1 / \lambda$. For this particular calculation, in order to obtain the specific result (1.3), we generalize to a slightly wider selection of jet sources than the ones used to simplify the general analysis of yet-higher-order corrections and fig. 1 .

Finally, we conclude in section VIII by pointing out a basic and still open problem about how $\ell_{\max } \propto E^{1 / 3}$ at strong coupling interpolates to $\ell_{\max } \propto E^{1 / 2}$ at small coupling.

\section{BASIC FRAMEWORK}

Our basic framework for studying jet stopping will be to create high-momentum excitations of the strongly-coupled plasma by perturbing the plasma with high-momentum sources, as in refs. [4, 5], and studying the response. As we will review below, for certain types of sources applied to the quantum field theory, the response in the gravity dual is the generation of a highly-localized and highly-oscillatory wave packet that moves through 


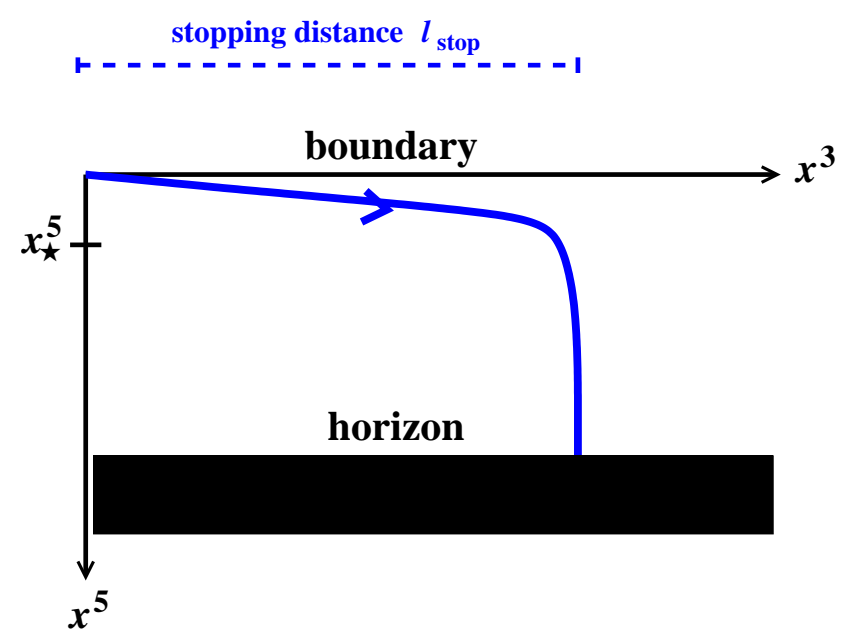

FIG. 3: Qualitative sketch of the motion of a particle (wave packet) through $\mathrm{AdS}_{5}$-Schwarzschild. As measured by $x^{0}$, the particle takes infinitely long to reach the horizon. Of special importance is the parametric scale $x_{\star}^{5}$ in the fifth dimension, where the trajectory turns over and beyond which progress in $x^{3}$ rapidly slows to a stop. Given our specific choice (2.1) of coordinates, we often refer to $x_{\star}^{5}$ as $z_{\star}$.

space while falling in the fifth dimension towards the black brane horizon. This wave packet has approximately well-defined 5-dimensional position and momentum, and (for $\lambda=\infty$ ), its motion can be approximated (up to parametrically small corrections) by a geodesic - that is, by the trajectory that a particle would take through the $\mathrm{AdS}_{5}$-Schwarzschild background. This particle (geometric optics) approximation makes the $\lambda=\infty$ calculation of stopping distances particularly simple and efficient. We will take the 3-momentum of our excitations to be in the $x^{3}$ direction, and the stopping distance is simply given by how far in $x^{3}$ the corresponding geodesic travels before falling into the black brane horizon, as depicted in fig. $3{ }^{6}$

We will later study the effect of higher-derivative corrections to the supergravity action by studying their effects on trajectories such as fig. 3. One effect is the change that higherderivative corrections make to the $\mathrm{AdS}_{5}$-Schwarzschild background, but we will see that the dominant effects are the changes they make to the equation of motion of the wave packets, which will no longer follow geodesics.

\section{A. Notation}

In this paper, we will use Greek letters for 4-dimensional space-time indices $(\mu, \nu=$ $0,1,2,3)$. Lower-case roman letters $(a, b)$ will be used for 10 -dimensional indices. The first five of those 10 dimensions, corresponding to $\mathrm{AdS}_{5}$-Schwarzschild when $\lambda=\infty$, will be represented by upper-case roman letters $(I, J=0,1,2,3,5)$. The remaining five dimensions,

6 For more discussion of why the distance the excitation travels before falling into the horizon should be identified with the stopping distance in the $3+1$ dimensional field theory problem, see the discussion in ref. [5], as well as earlier discussions in the context of falling classical strings [1, 19] 
corresponding to the compact 5 -sphere $S^{5}$, will be indicated by dotted lower-case roman letters $(\dot{a}, \dot{b})$. When we use the adjective "five-dimensional" without further qualification, then we are referring to the first 5 dimensions - those of $\mathrm{AdS}_{5}$-Schwarzschild.

The form of the metric we will use for $\mathrm{AdS}_{5}-$ Schwarzschild is $^{7}$

$$
(d s)^{2}=\frac{\mathrm{R}^{2}}{z^{2}}\left[-f(d t)^{2}+(d \boldsymbol{x})^{2}+f^{-1}(d z)^{2}\right]
$$

where $z$ is the coordinate $x^{5}$ of the fifth dimension, $\mathrm{R}$ is the radius of the 5 -sphere (and will drop out of final results),

$$
f \equiv 1-\frac{z^{4}}{z_{\mathrm{h}}^{4}}
$$

the boundary is at $z=0$, and the horizon is at

$$
z_{\mathrm{h}}=\frac{1}{\pi T} .
$$

We will not need to worry about the details of regularizing the location of the boundary in this work. ${ }^{8}$

The metric of 4-dimensional flat space-time will be represented by $\eta_{\mu \nu} \equiv$ $\operatorname{diag}(-1,+1,+1,+1)$.

\section{B. Review of $\lambda=\infty$ results}

\section{Set up}

In this paper, we follow the approach of refs. [4, 5] for studying the jet stopping problem. An external source is applied to the strongly-interacting gauge theory in order to create the initial high-energy high-momentum excitation. Specifically, we add a source term to the Lagrangian,

$$
\mathcal{L} \rightarrow \mathcal{L}+\mathcal{N} O(x) e^{i \bar{k} \cdot x} \Lambda_{L}(x)
$$

where $\mathcal{N}$ is an arbitrarily small source amplitude, $O(x)$ is a source operator,

$$
\bar{k}^{\mu} \simeq(E, 0,0, E)
$$

is the large 4-momentum of the desired excitation, and $\Lambda_{L}(x)$ is a slowly varying envelope function that localizes the source to within a distance $L$ of the origin in both $x^{3}$ and time. For example,

$$
\Lambda_{L}(x)=e^{-\frac{1}{2}\left(x^{0} / L\right)^{2}} e^{-\frac{1}{2}\left(x^{3} / L\right)^{2}} .
$$

$L$ is chosen large compared to $1 / E$ but small compared to the stopping distance we wish to measure. The small amplitude $\mathcal{N}$ is so that we can treat the external source as a smallperturbation to the strongly-interacting gauge theory, so that the source will never create more than one jet with energy $E$ at a time.

7 The coordinate used in refs. [4, [5] is $u=z^{2} / z_{\mathrm{h}}^{2}$, which is $u=(z / 2)^{2}$ when working in the units $2 \pi T=1$ used there.

8 See section $\mathrm{V}$ of ref. [5] for some discussion of boundary regularization in the context of making the particle approximation. 
The source operator $O(x)$ is a matter of choice. As an example, ref. [4] found it convenient to focus on "jets" created by an external R-charge field (somewhat analogous to the excitation that would be created by the hadronic decay of a high-momentum $\mathrm{W}$ boson inside a standard-model quark-gluon plasma, but with isospin replaced by $\mathrm{R}$ charge). In that case the operator was $O(x)=j^{\perp}(x)$, where $j^{\mu}$ is a combination of $\mathrm{R}$ current operators. The details of the choice of source operator $O$ are unimportant [5], however, as long as the operator has finite conformal dimension in the $\lambda=\infty$ limit. We'll later discuss the choice we find convenient for the present study of finite- $\lambda$ corrections.

Sometimes in previous work [4], the characteristic 4-momentum $\bar{k}$ of the source has been taken to be exactly light-like, $\bar{k}=(E, 0,0, E)$. Because the source is confined to a space-time region of size $L$, the momentum components $q^{\mu}$ of the source are smeared out around $\bar{k}^{\mu}$ by an amount of order $1 / L$, and so the typical magnitude of the virtuality $q^{2} \equiv q^{\mu} q_{\mu}$ of the source is then of order $\left|q^{2}\right| \sim E / L \ll E^{2}$.

In later work, a calculational and conceptual simplification was found if one instead chooses the characteristic 4 -momentum $\bar{k}$ to be just a little bit time-like,

$$
\bar{k}=(E+\epsilon, 0,0, E-\epsilon)
$$

with

$$
\frac{1}{L} \ll \epsilon \ll E
$$

The first inequality guarantees that the uncertainty in momentum does not overwhelm the size of $\epsilon$. In this case, the source has an approximately well-defined virtuality in 4-momentum space $q$ of

$$
-q^{2} \equiv-q^{\mu} q_{\mu} \simeq-\bar{k}^{\mu} \bar{k}_{\mu} \simeq 4 \epsilon E
$$

This is the case where the response created in the dual gravity theory can be shown [5] to be a highly localized, highly oscillatory wave packet that falls in the fifth dimension toward the black brane horizon. The trajectory of the wave packet is the geodesic that would be followed by a massless 5-dimensional particle traveling in the $\mathrm{AdS}_{5}$-Schwarzschild background as in fig. 3. Calculations using this particle picture [5] are much simpler and more efficient than calculations directly in terms of the 5-dimensional field excitations [4].

\section{The geodesic}

The 5-dimensional mass $m$ associated with a supergravity field, and therefore with the 5 -dimensional particle trajectory, is determined by the conformal dimension $\Delta$ of the field theory operator dual to that field. ${ }^{9}$ We will take $\Delta$ to be of order one. It was shown in ref. [5] that, in the high-energy limit, this mass does not affect the stopping distance for sources described by (2.7) when $\ell_{\text {stop }} \ll \ell_{\max }$, which will be our focus here. So we may ignore the 5-dimensional mass and focus on the trajectories $d x^{I} d x_{I}=0$ corresponding to null geodesics in $\mathrm{AdS}_{5}$-Schwarzschild. The solution for such geodesics (for a metric that depends only on

9 It is important to note that the masses of 5-dimensional fields in the gravity dual have nothing to do with the masses of 4 -dimensional excitations in the $\mathcal{N}=4 \mathrm{SYM}$ field theory. The 5-dimensional mass $m$ is not the "mass of a jet." 
$x^{5}$ and has 4-dimensional parity) is

$$
x^{\mu}\left(x^{5}\right)=\int \sqrt{g_{55}} d x^{5} \frac{g^{\mu \nu} q_{\nu}}{\left(-q_{\alpha} g^{\alpha \beta} q_{\beta}\right)^{1 / 2}},
$$

where the 4-momentum $q_{\alpha}$ with lower index is conserved in 5-dimensional motion and is given by the 4-momentum (2.7) of our source, $q_{\alpha}=\eta_{\alpha \beta} \bar{k}^{\beta}$.

Taking the integral in (2.9) all the way to the horizon for $\mu=3$ (the direction of the jet), and using the metric (2.1), gives the geometric optics approximation to the stopping distance

$$
\ell_{\text {stop }} \simeq \int_{0}^{z_{\mathrm{h}}} d z \frac{|\boldsymbol{q}|}{\sqrt{-q^{2}+\frac{z^{4}}{z_{\mathrm{h}}^{4}}|\boldsymbol{q}|^{2}}}
$$

where we have used rotation invariance to rewrite $q_{3}$ as $|\boldsymbol{q}|$. Here and throughout this paper we use the symbol $q^{2}$ for the 4 -virtuality of the source,

$$
q^{2} \equiv q_{\mu} \eta^{\mu \nu} q_{\nu}<0
$$

Throughout this paper, we will restrict attention to the case $-q^{2} \ll E^{2}$ as in (2.8), as this is the case which generates stopping distances large compared to $1 / T$. In this limit, the integral in (2.10) is dominated by small values of $z$, of order

$$
z_{\star} \sim z_{\mathrm{h}}\left(\frac{-q^{2}}{|\boldsymbol{q}|^{2}}\right)^{1 / 4} \sim z_{\mathrm{h}}\left(\frac{-q^{2}}{E^{2}}\right)^{1 / 4} \ll z_{\mathrm{h}},
$$

corresponding to the parametric scale labeled $x_{\star}^{5}$ in fig. 3. Neglecting parametrically small corrections, we may replace the upper limit of integration by infinity in (2.10) to get $^{10}$

$$
\ell_{\text {stop }} \simeq \frac{\Gamma^{2}\left(\frac{1}{4}\right)}{(4 \pi)^{1 / 2}}\left(\frac{E^{2}}{-q^{2}}\right)^{1 / 4} \frac{1}{2 \pi T} .
$$

The validity of (2.13) is restricted to the range of validity of the geometric optics approximation. For a detailed discussion, see ref. [5]. Here, for the sake of simplicity of this review, we will just give a quick, crude way to see the limit of applicability from the result (2.13) itself. By the uncertainty principle, the components of the source's 4-momentum will be smeared out by $1 / L$, where $L$ is the source size. Consequently, the virtuality $-q^{2}$ given by (2.8) will only be (approximately) well-defined when $\epsilon \gg 1 / L$ and so when

$$
-q^{2} \gg \frac{E}{L}
$$

But the result (2.13) from the geometric optics approximation is only meaningful if $-q^{2}$ is approximately well defined. Combining (2.13) and (2.14) requires

$$
\frac{\ell_{\text {stop }}^{4} T^{4}}{E} \ll L
$$

$\overline{10}$ See ref. [5] for this explicit result, but the parametric behavior $\ell_{\text {stop }} \sim\left(E^{2} /-q^{2}\right)^{1 / 4}$, within its range of validity, was found earlier by Hatta, Iancu and Mueller [2]. 
On the other hand, it wouldn't be sensible to try to measure a stopping distance unless we choose a source size that is smaller than the distance we wish to measure. So $L$ needs to satisfy

$$
\frac{\ell_{\text {stop }}^{4} T^{4}}{E} \ll L \ll \ell_{\text {stop }} .
$$

Choosing such an $L$ is possible exactly when $\ell_{\text {stop }} \ll \ell_{\max }$ with $\ell_{\max }$ given by (1.1).

The chance of propagating excitations created by sources like (2.4) to distances $\gg \ell_{\max }$ is negligible. But showing this convincingly requires abandoning the geometric optics analysis and doing a wave analysis, as in refs. [2, 4]. We leave that to section VII. For most of this paper, we will stick to the region $\ell_{\text {stop }} \ll \ell_{\max }$ where the geometric optics approximation is valid, and then make only parametric extrapolations to the boundary $\ell_{\text {stop }} \sim \ell_{\max }$ of the range of validity.

We should also mention that even for $\ell_{\text {stop }} \ll \ell_{\max }$, the geometric approximation eventually breaks down at sufficiently large $z \gg z_{\star}$. At that point, however, the wave packet is falling essentially straight down towards the horizon, and the fact that it can no longer be treated as a particle no longer matters to how far it travels in $x^{3}$. The stopping distance is determined by the behavior of the trajectory for $z \sim z_{\star}$. (That is, $z \ll z_{\star}$ and $z \gg z_{\star}$ give parametrically small contributions to the stopping length.)

\section{Other authors' methods for describing "jets"}

Let's pause a moment to compare and contrast the type of jets we create with some of the others studied in the literature. Our formalism [4, 5] (with and without the particle approximation) can be thought of as a concrete way to realize early ideas of Hatta, Iancu and Mueller [2].

Alternatively, there is a long history of considering jet-like states that are dual to classical strings falling towards the horizon in the gravity theory (as well as a history of using geodesics to help understand the strings' motion) [1-3]. One difference is that the maximum stopping distance for these states is parametrically smaller than for the states we consider $-\ell_{\max }$ for the states related to classical strings scales as $\lambda^{-1 / 6} E^{1 / 3} T^{-4 / 3}$ rather than the $E^{1 / 3} T^{-4 / 3}$ of (1.1). It's amusing to note that, perhaps coincidentally, $\lambda^{-1 / 6} E^{1 / 3} T^{4 / 3}$ is the same stopping distance scale where corrections become problematical in our fig. 1. In any case, we will not attempt here to study $1 / \lambda$ corrections to previous results based on classical strings.

Yet another, recent method for creating a gluon-like jet is to generate it as a beam of synchrotron radiation from a heavy quark that is forced into circular motion [6]. These gluon-like jets (under certain conditions) penetrate a distance of order $\ell_{\max }$ given by (1.1). We will not attempt to study the $1 / \lambda$ corrections in this synchrotron problem, but we would not be surprised if they work out similar to the $\ell_{\text {stop }} \sim \ell_{\max }$ case in our analysis.

Finally, since coupling does not run with scale in $\mathcal{N}=4$ SYM, we are treating the coupling as large at all scales relevant to energy loss. This is in contrast to programs, such as ref. [14], that try to isolate the soft effects of a strongly-coupled medium on weakly-coupled hard bremsstrahlung or pair-production vertices. ${ }^{11}$ For work on $1 / \lambda$ corrections in that context, see ref. [16].

11 For a very brief summary of the relevant scales for the coupling, see, for example, ref. 15. 


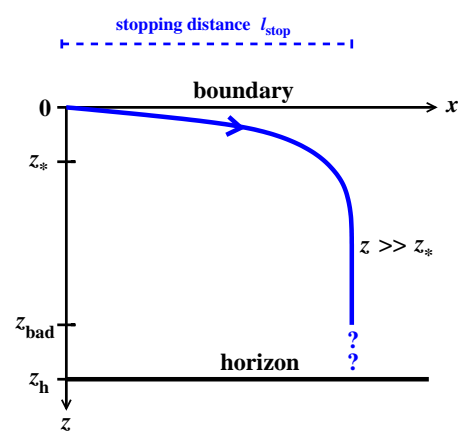

(a)

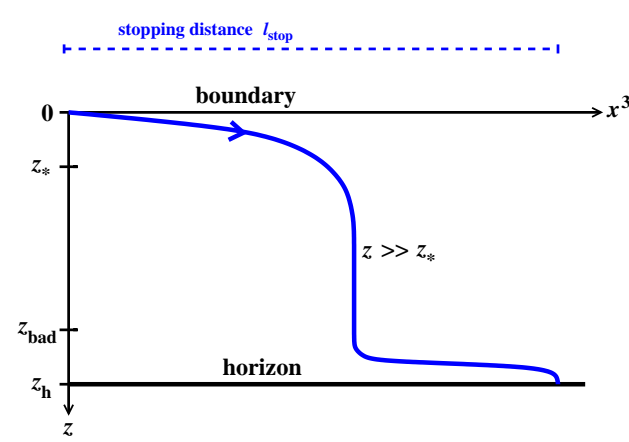

(b)

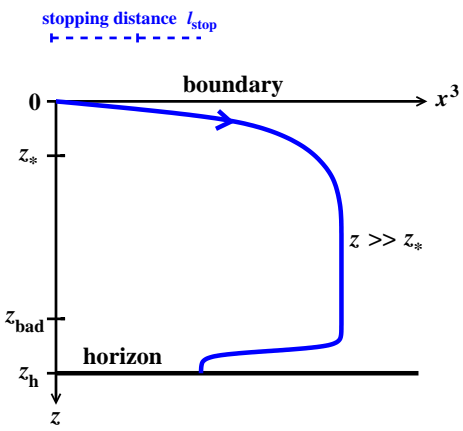

(c)

FIG. 4: (a) A picture of the trajectory of the 5-dimensional wave packet when higher-derivative corrections have negligible effect on the trajectory at $z \sim z_{\star}$. The trajectory stops moving in $x^{3}$ as $z$ increases well above $z_{\star}$, falls straight toward the horizon, and then eventually reaches a large enough $z$ (labeled $z_{\text {bad }}$ ) that the expansion in higher-derivative effects breaks down. (b,c) What we assume does not happen. In all of these figures, only the parametric ordering $z_{\star} \ll z_{\text {bad }}$ is significant: $z_{\text {bad }}$ is not necessarily close to the horizon.

\section{Determining the importance of corrections}

We wish to check whether or not higher-derivative corrections to the supergravity action can invalidate the $\lambda=\infty$ result for finite but large $\lambda$ and large energy. We have seen above that the $\lambda=\infty$ stopping distance is generated by the behavior of the particle trajectory for $z \sim z_{\star}$ given by (2.12). So the simple way to address our question is to check whether or not higher-derivative corrections make significant changes to the trajectory for $z \sim z_{\star}$. The "importance" represented by the vertical axis of fig. 1 is just the relative effect on the trajectory at $z \sim z_{\star}$.

We will see later in section VI that the relative effects of higher-derivative corrections increase with increasing $z$. We will see that, at sufficiently high energies, there is always a point $z \gg z_{\star}$ where, in the geometric optics approximation, the expansion in effects of higher-derivative corrections goes bad. In some cases, this will occur for z's large enough that the geometric optics approximation has already broken down there anyway. But in all cases, we will make the following, physically reasonable assumption in our analysis:

Assumption 1. Once the 5-dimensional wave packet has stopped moving significantly in $x^{3}$, so that it is falling essentially straight toward the horizon, then it will thereafter continue falling essentially straight toward the horizon and will not move significantly in $x^{3}$ again.

That is, if the wave packet's trajectory looks like fig. 4a, we will assume that it does not actually behave like figs. 4b or c.

There is an additional assumption in our analysis. We will read off the jet stopping distance to be the distance $x^{3}$ that the 5 -dimensional wave packet travels before it falls into the horizon. But really, one should discuss the stopping distance directly in terms of observables in the boundary theory. Following Chesler et al. [19], we could look at the location of the source terms for late-time hydrodynamic diffusion or sound propagation of the charge and energy and momentum densities, deposited by the jet in the medium once the jet stops and thermalizes. For simplicity, consider the case of jets that carry $\mathrm{R}$ 


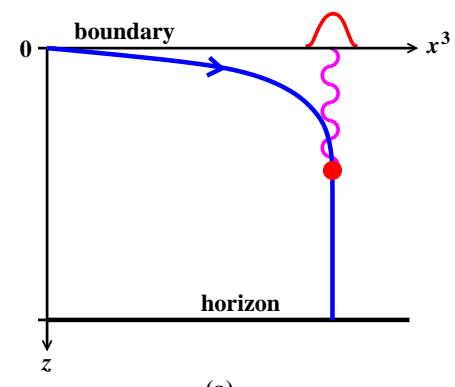

(a)

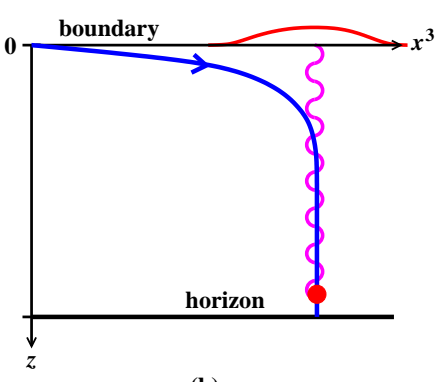

(b)

FIG. 5: As the wave packet falls towards the horizon, the effects of its $\mathrm{R}$ charge on the boundary theory redshifts, corresponding to a hydrodynamically diffusing charge density measured in the 4-dimensional plasma.

charge. The presence of the wave packet in the bulk produces a response in the boundary fields that are dual to $\mathrm{R}$ charge, as depicted in fig. $5 \mathrm{a}$. As the 5-dimensional wave packet approaches the horizon, this disturbance is further and further red-shifted, which corresponds to hydrodynamic diffusion of the charge density in the boundary theory, as depicted at later times in fig. 5b. In the $\lambda=\infty$ calculation, the charge distribution is centered in $x^{3}$ over the position of the wave packet as it approaches the horizon. For the particular type of jets we study in this paper, more detail may be found by comparing refs. [4] and [5], but the same type of behavior occurs in earlier works on jets dual to classical strings [1, 3, 19]. Our assumption will be that this correspondence continues for high-energy jets at large but finite $\lambda$ :

Assumption 2. $1 / \lambda$ corrections do not significantly modify the (approximate) equality between (i) the late-time $x^{3}$ position of the 5 -dimensional wave packet as it approaches the horizon and (ii) the position where the jet stops and thermalizes in the 4-dimensional field theory as measured, for example, by the center of the late-time diffusing distribution of $\mathrm{R}$ charge.

In particular, in this paper we will not attempt to make a thorough analysis of $1 / \lambda$ corrections to the coupling between the 5-dimensional wave packet and the 5-dimensional gauge field bulk-to-boundary propagator associated with making a late-time measurement of the $\mathrm{R}$ charge distribution. ${ }^{12}$ (Nor will we make the corresponding analysis for the coupling between the wave packet and the graviton propagator associated with making a measurement of the

12 As discussed in ref. [4], the bulk-to-boundary propagator associated with the late-time measurement is a low-momentum propagator. This propagator therefore contains no powers of large energy $E$ that could compensate powers of $1 / \lambda$, and so the corrections to that propagator are always small for large finite $\lambda$. When one extracts from the hydrodynamic response the place in the plasma where the charge was deposited, by applying the 4-dimensional diffusion operator $\left(\partial_{t}-D_{R} \boldsymbol{\nabla}^{2}\right)$ as in refs. [4, 19], the 5 -dimensional bulk-to-boundary propagator just discussed gets truncated and only has support at the 4-position of the bulk vertex. (See, for example, the related discussion in sec. III.D of ref. [17].) Formally, that means that charge deposition only has support for 4-positions traveled through by the high-energy 5 -dimensional wave packet created by the source. One might think this guarantees the validity of our Assumption 2. However, it is possible for a series of local functions to add up to something with support 
sound waves produced by the jet.) We will only analyze corrections to how far the 5dimensional wave packet travels.

\section{Our choice of source operator}

The source operator $O(x)$ used to generate the jet via (2.4) determines the type of field that is excited in the dual theory - that is, the type of wave packet whose trajectory is depicted by fig. 3. To keep our analysis simple, we will choose a class of source operators that are dual to fields which are 5-dimensional scalars after the $S^{5}$ reduction. (We will slightly relax this restriction later, in section VII, when presenting an example of an explicit calculation of a leading correction.) Moreover, in 10-dimensional language, it will be convenient to mainly focus on the purely gravitational terms in the supergravity action (about which the most is known). So we will pick our 5-dimensional scalar field $\phi$ from the components $h_{\dot{a} \dot{b}}$ of the 10-dimensional metric fluctuations $h_{a b}$ with indices $\dot{a}, \dot{b}$ along the 5 -sphere $S^{5}$. Finally, it will be convenient to only consider the traceless parts of $h_{\dot{a} \dot{b}}$ to avoid mixing with other supergravity fields. ${ }^{13}$ Other than these constraints, the Kaluza-Klein mode may be whatever one desires, and will determine, for example, the $\mathrm{R}$ charge of the excitation. In summary, we shall consider a 10-dimensional field of the form

$$
h_{a b}(x, y)= \begin{cases}\phi(x) Y_{a b}(y), & a, b \in\{6,7,8,9,10\} \\ 0, & \text { otherwise }\end{cases}
$$

where $x$ is the $\mathrm{AdS}_{5}$-Schwarzschild coordinate, $y$ is the $S^{5}$ coordinate, $\phi$ is a 5 -dimensional scalar field, and $Y_{\dot{a} \dot{b}}(y)$ is any traceless tensor spherical harmonic on $S^{5}$. We will crudely summarize this choice by writing the field as $h_{\dot{a} \dot{b}}$ when thinking of it as a 10-dimensional field and as $\phi$ when thinking of it as a 5-dimensional field.

Source operators dual to the fields (2.17) are those operators of the form ${ }^{14}$

$$
O \sim \operatorname{tr}\left(\lambda \lambda \bar{\lambda} \bar{\lambda} X^{k}\right), \quad k=0,1,2, \cdots
$$

obtainable as supersymmetry descendants $Q^{2} \bar{Q}^{2}$ of $\operatorname{tr}\left(X^{k+4}\right)$, where the $X$ are the adjointcolor scalar fields of $\mathcal{N}=4 \mathrm{SYM}$ and $\lambda$ are the gluinos. The source operators (2.18) have conformal dimension $\Delta=k+6$ and carry non-trivial $\mathrm{R}$ charge (and so the jets they produce will carry $\mathrm{R}$ charge). Different choices $k$ in (2.18) produce different representations $(2, k, 2)$ of the $\mathrm{SU}(4) \mathrm{R}$ symmetry, which correspond to different types of tensor harmonics $Y_{\dot{a} \dot{b}}(y)$ in (2.17). None of the details of $\mathrm{R}$ charge representations, which element we choose, or tensor harmonics will matter for what follows.

elsewhere, as in $\sum_{n}\left(a^{n} / n !\right) d^{n} \delta(x) / d x^{n}=\delta(x+a)$. Our Assumption 2 is that at late times (when the wave packet is very near the horizon), $1 / \lambda$ corrections to the bulk 3 -point vertex do not somehow sum up in a similar way to produce a large percentage change to the stopping distance.

13 For example, the trace $h_{\dot{a}}^{\dot{a}}$ over $S^{5}$ indices mixes with fluctuations of the 5 -form field strength $F_{\dot{a} \dot{b} \dot{c} \dot{d} \dot{e}}$ on $S^{5}[24]$.

14 See, for example, Table 7 of the review by D'Hoker and Freedman [23]. Here $X^{k}$ is shorthand for any symmetric product $X^{\left(i_{1}\right.} X^{i_{2}} \cdots X^{\left.i_{k}\right)}$ of $k$ factors of the three complex scalar fields $X^{1}, X^{2}, X^{3}$ of $\mathcal{N}=4$ SYM. 
It would be useful to check that our qualitative conclusions are not sensitive to picking this particular class of source operators. But, for most of this paper, we will simply stick to fields (2.17) in the gravity dual and so source operators (2.18). However, in studying the leading corrections to exponential tails in section VII, we will make a slight generalization to also consider the field $h_{12}$, dual to the $T^{12}$ component of the stress tensor.

Throughout this paper, we will for simplicity treat the conformal dimension $\Delta$ of the source operator as parametrically of order one, $\Delta \sim 1$. See ref. [5] for a discussion in the context of $\lambda=\infty$ stopping distances of what happens when $\Delta \gg 1$ but with $\Delta$ still parametrically small compared to powers of $E$ and $\lambda$.

\section{THE $R^{4}$ CORRECTION}

We now want to discuss how higher-derivative corrections to the supergravity action affect the 5-dimensional particle equations of motion. It will be helpful to do this first in the context of a concrete example of a higher-derivative correction. Though our final qualitative conclusions will not depend on the exact pattern of higher-derivative supergravity corrections that arise from Type IIB string theory, it will be convenient to start the discussion with the first one that does, ${ }^{15}$ which is $R^{4}$.

\section{A. $\quad R^{4}$ term in the 10 -dimensional supergravity action}

The first corrections to low-energy supergravity arise from the low-energy limit of the string-string scattering amplitude, for which $N_{\mathrm{c}}=\infty$ corresponds to the tree-level amplitude. The string-string scattering amplitude with 4 external graviton states as legs gives rise to an $R^{4}$ interaction in 10-dimensional supergravity, where one may crudely think of each factor of $R$ as corresponding to each leg. Through use of the equations of motion, one may in fact put the gravitational interaction in the form $C^{4}$ where $C$ is the Weyl tensor. (We will not need to make such "on-shell" assumptions about higher-derivative corrections in our general analysis later on.) The details of how the four Weyl tensors are contracted will not matter to our parametric analysis but, for the sake of concreteness, here is the explicit modification to the low-energy supergravity action: ${ }^{16}$

$$
R \rightarrow R+\frac{1}{8} \zeta(3) \alpha^{\prime 3}\left[C^{h m n k} C_{p m n q} C_{h}^{r s p} C^{q}{ }_{r s k}+\frac{1}{2} C^{h k m n} C_{p q m n} C_{h}{ }^{r s p} C^{q}{ }_{r s k}\right]
$$

or equivalently (via Bianchi identities)

$$
R \rightarrow R+\frac{1}{8} \zeta(3) \alpha^{\prime 3}\left[C^{h m n k} C_{p m n q} C_{h}^{r s p} C_{k r s}^{q}+\frac{1}{4} C^{h k m n} C_{p q m n} C_{h}^{p r s} C^{q}{ }_{k r s}\right]
$$

15 For readers who are instead curious about what would happen in Gauss-Bonnet gravity, see Appendix C3.

16 Eq. (3.1) is nicely summarized in eqs. (3.1-3) of ref. 25] and originates from refs. 22, 26]. We have fixed the dilaton field $\varphi$ since we will not need to consider its fluctuations. (When comparing to more general results involving modular forms depending on $\varphi$, note that the $N_{\mathrm{c}} \rightarrow \infty$ limit corresponds to the large $\varphi$ limit. After taking the large $\varphi$ limit, one may rescale away any remaining dependence on the constant value of $\varphi$.) 
where $R$ is the Ricci scalar in this equation, and $\alpha^{\prime}$ is the string tension. The usual duality relation between the string tension and 't Hooft coupling is [27]

$$
\frac{\alpha^{\prime}}{\mathrm{R}^{2}}=\lambda^{-1 / 2} \equiv\left(g_{\mathrm{YM}}^{2} N_{\mathrm{c}}\right)^{-1 / 2},
$$

where $\mathrm{R}$ is the $S^{5}$ radius.

\section{B. The $\phi$ equation of motion}

Let's now figure out the form of the corresponding linearized equation of motion for our 5-dimensional scalar field $\phi \sim h_{\dot{a} \dot{b}}$ in the $\mathrm{AdS}_{5}$-Schwarzschild background. By "linearized," we mean linearized in $\phi$, not in the background metric. That means that we want terms in the action (3.2) that are quadratic in $\phi$.

The $C^{4}$ correction term is suppressed by $\alpha^{3} \propto \lambda^{-3 / 2} \ll 1$, and so the only way we can get an unsuppressed correction is if there are compensating factors of the large energy $E$ associated with the $\phi$ wave packet created by the source. The dominant correction will be the one with the most powers of $E$. Powers of $E$ will arise from derivatives (with indices in $\mathrm{AdS}_{5}$-Schwarzschild) hitting $\phi$. So we should focus on the pieces of the $C^{4}$ term in the action (3.2) that are quadratic in $\phi$ and have as many 5-dimensional derivatives acting on $\phi$ as possible.

One way to get a factor of $\phi \sim h_{\dot{a} \dot{b}}$ is to consider the piece of the Weyl tensor $C_{i j k l}$ that involves two 5 -dimensional derivatives of the $S^{5}$ metric fluctuation $h_{\dot{a} \dot{b}}$, e.g.

$$
C_{I \dot{a} J \dot{b}} \simeq-\frac{1}{2} \nabla_{I} \nabla_{J} h_{\dot{a} \dot{b}}
$$

Other terms, with fewer derivatives acting on $h_{\dot{a} \dot{b}}$, will be suppressed because they do not generate as many factors of $E$. Similarly, a term involving $S^{5}$ derivatives like $\nabla_{\dot{m}} \nabla_{\dot{n}} h_{\dot{a} \dot{b}}$ arising from $C_{\dot{m} \dot{a} \dot{n} \dot{b}}$ will be suppressed compared to (3.4) because $S^{5}$ derivatives of $\phi$ do not yield factors of $E$. The dominant terms in $C^{4}$ that contribute to the linearized $\phi$ equation of motion will therefore be those terms that have two factors of the form (3.4) and two factors of the background Weyl tensor $\bar{C}_{i j k l}$ of $\left(\mathrm{AdS}_{5}\right.$-Schwarzschild $) \times S^{5}$. Now use the fact that the background Weyl tensor $\bar{C}_{i j k l}$ in this case vanishes unless all of its indices live in $\mathrm{AdS}_{5}$-Schwarzschild. ${ }^{17}$ The dominant $C^{4}$ terms in the action then have the form

$$
\# \alpha^{\prime 3}(\nabla \nabla \phi)(\nabla \nabla \phi) \bar{C} \bar{C}
$$

where \# indicates some coefficient and the suppressed indices on $\nabla$ and $\bar{C}$ are all fivedimensional indices and contracted.

Note that such terms only arise from the first term in brackets in (3.2) and not the second. For $\frac{1}{4} C^{h k m n} C_{p q m n} C_{h}{ }^{p r s} C^{q}{ }_{k r s}$, getting two factors of the form (3.4) would require evaluating background Weyl tensors $\bar{C}$ with at least one $S^{5}$ index, which gives zero.

17 This is a statement about the uncorrected, i.e. $\lambda=\infty,\left(\mathrm{AdS}_{5}\right.$-Schwarzschild $) \times S^{5}$ background, and does not account for corrections to that background due to $\alpha^{\prime 3} C^{4}$. But, as we shall see, this is good enough for figuring out the leading correction to the $\phi$ equation of motion. 
The 5-dimensional equation of motion for $\phi$ corresponding to (3.5) has the schematic form $^{18}$

$$
\left[\nabla \nabla+\#\left(\alpha^{\prime}\right)^{3} \nabla \nabla \bar{C} \bar{C} \nabla \nabla\right] \phi=0,
$$

where we have dropped the 5-dimensional mass term (determined by the conformal dimension of the source operator and arising in part from $S^{5}$ derivatives on $\phi$ in the leading-order supergravity action). As mentioned earlier, the mass is ignorable when computing the stopping distance for $\ell_{\text {stop }} \ll \ell_{\max }$.

\section{The WKB approximation}

Now let's look for wave solutions to the 5-dimensional equation of motion (3.6) that have large, definite 4-momentum $q_{\mu}$ :

$$
\phi=\Phi\left(x^{5}\right) e^{i q_{\alpha} x^{\alpha}} .
$$

(We will superpose such solutions to make wave packets in the next section.) Remember that Greek indices run over 4-dimensional space-time. At high energy, we can make a WKB-like approximation and re-write (3.7) as

$$
\phi=e^{i S\left(x^{5}\right)} e^{i q_{\alpha} x^{\alpha}}
$$

where $S\left(x^{5}\right)$ is large. As discussed in refs. [4, 5], this approximation only works sufficiently far from the boundary: $z \gg z_{\mathrm{WKB}}$ where $z_{\mathrm{WKB}} \sim 1 / \sqrt{-q^{2}}$. But for $\ell_{\text {stop }} \ll \ell_{\max }$ (i.e. $\left.-q^{2} \gg E^{2 / 3} T^{4 / 3}\right)$ that is good enough to analyze the stopping distance, which is dominated by $z \sim z_{\star}$ given by (2.12).

Now use (3.8) in the equation of motion (3.6). 4-space derivatives $\nabla_{\mu}$ will give the largest contribution when they hit the phase factor in (3.8) and bring down a factor of the large $q_{\alpha}$ rather than hitting something involving the background metric. $x^{5}$ derivatives $\nabla_{5}$ will give the largest contribution when they hit the $e^{i S\left(x^{5}\right)}$ in $(3.8)$ and bring down a large factor of $i \partial_{5} S$ rather than hitting something involving the background metric. So, in the large-energy limit, the dominant terms correspond to replacing

$$
\nabla_{I} \rightarrow i Q_{I} \equiv i\left(q_{\mu}, q_{5}\right)
$$

in the equation of motion for $\phi$, where

$$
q_{5} \equiv \frac{\partial S}{\partial x^{5}}
$$

We capitalize $Q_{I}$ just as a way of notationally emphasizing that it is a 5 -vector momentum. The result of (3.9) is to replace the equation of motion (3.6) by

$$
\left[-Q Q+\# \alpha^{\prime 3} Q Q \bar{C} \bar{C} Q Q\right] \phi=0
$$

18 For readers wishing to compare (i) the schematic equation (3.6) that summarizes the dominant terms in the high energy limit to (ii) the full, explicit equation of motion for a specific example (the bottom of the Kaluza-Klein tower for traceless $h_{\dot{a} \dot{b}}$ ), see Appendix $\mathrm{A}$ 
One may then solve this equation algebraically for $q_{5}$ as a function of $q_{\mu}$ and $x^{5}$. Integrating (3.10) then gives ${ }^{19}$

$$
\phi \simeq e^{i q_{\alpha} x^{\alpha}+i \int q_{5} d x^{5}}
$$

where $q_{5}=q_{5}\left(q_{\mu}, x^{5}\right)$ solves (3.11).

Just for the sake of being concrete, we give the explicit formula for the equation of motion (3.11) for the explicit $C^{4}$ operator given by (3.2):

$$
-(\bar{g}+\delta g)^{Q S} Q_{Q} Q_{S}+\frac{1}{4} \zeta(3) \alpha^{\prime 3} \overline{Q^{H} Q^{K} Q_{P} Q_{Q} C_{H}{ }^{R S P} C^{Q}{ }_{R S K}} \simeq 0,
$$

where overlines indicate that the 5 -dimensional metric is taken to be $\mathrm{AdS}_{5}$-Schwarzschild (2.1), and $\delta g$ is the correction to that metric caused by the $C^{4}$ operator.

We do not need to work out $\delta g$. It is enough to note that this distortion $\delta g$ of the equilibrium metric has nothing to do with the fact that we have injected the $\phi$ wave packet into the system and so is independent of $E$. Its effects are therefore suppressed by powers of $\lambda^{-1 / 2}$ with no compensating factors of $E .^{20}$ So we will drop $\delta g$ and no longer bother explicitly writing the overlines to indicate the $\mathrm{AdS}_{5}$-Schwarzschild metric:

$$
-Q^{I} Q_{I}+\frac{1}{4} \zeta(3) \alpha^{\prime 3} Q^{H} Q^{K} Q_{P} Q_{Q} C_{H}{ }^{R S P} C^{Q}{ }_{R S K} \simeq 0
$$

We will discuss in a moment the parametric size of the $\alpha^{\prime 3}$ correction to the $\lambda=\infty$ dispersion relation $Q^{I} Q_{I} \simeq 0$. But first, we will briefly review how to turn a dispersion relation like (3.14) into a particle trajectory using the geometric optics approximation.

\section{The geometric optics (particle) approximation}

The geometric optics approximation consists of approximating the wave packets as simultaneously having (i) well defined 5-momentum $Q_{I}$, satisfying (3.14) above, and (ii) well defined 5-position $\left(x^{\mu}, x^{5}\right)$. One way to get the particle equation of motion is to start from the WKB approximation (3.12) to the boundary-to-bulk propagator and to make a wave packet by convolving with an appropriate localized boundary source function $\Lambda_{L}(x)$ :

$$
\phi(x) \sim \int d^{4} q e^{i q_{\alpha} x^{\alpha}+i \int q_{5}\left(q, x^{5}\right) d x^{5}} \tilde{\Lambda}_{L}(-q) .
$$

This integral can be done by saddle point methods, and the saddle point condition is

$$
0=\frac{\partial}{\partial q_{\mu}}\left[i q_{\alpha} x^{\alpha}+i \int q_{5}\left(q, x^{5}\right) d x^{5}\right]
$$

19 There is a slight difference in sign convention between our work here and in some previous work by two of the authors. In ref. [4], $q_{\mu}$ was the 4-momentum conjugate to the boundary (rather than bulk) position, which would be $-q_{\mu}$ in the convention used in (3.8) and (3.12). With the convention used in our current paper, retarded boundary-to-bulk propagators (also known as advanced bulk-to-boundary propagators) correspond to solutions with $q_{5}>0$.

20 One should pause to consider whether $\delta g$ might blow up faster than $\bar{g}$ at small $z$, since powers of $1 / z$ do give powers of $E$ at the $z$ scale $z_{\star}$ of interest (2.12). However, as one takes $z \rightarrow 0, \mathrm{AdS}_{5}$-Schwarzschild approaches AdS, and it is known that higher-derivative corrections do not modify the AdS solution [28]. This means that the modifications to $\mathrm{AdS}_{5}$-Schwarzschild will not become large as $z \rightarrow 0$. 
which gives

$$
x^{\mu}=-\int d x^{5} \frac{\partial q_{5}}{\partial q_{\mu}} .
$$

Formally, we may then use this expression to find the generalization

$$
\ell_{\text {stop }} \simeq-\int_{0}^{z_{\mathrm{h}}} d z \frac{\partial q_{5}}{\partial|\boldsymbol{q}|}
$$

of the stopping distance integral (2.10). We will see later that this integral will require care in interpretation in the region where $z$ is large enough that the expansion in higherderivative corrections breaks down, as in fig. 4. Our focus will be on the relative importance of higher-derivative corrections in the integrand at $z \sim z_{\star}$.

See ref. [5] for a $\lambda=\infty$ discussion of when the wave packet is small enough to treat as a particle. The summary is that $L$ can be chosen appropriately so that everything is fine at $z \sim z_{\star}$ when $\ell_{\text {stop }} \ll \ell_{\text {max }}$.

\section{E. The relative importance of the $C^{4}$ correction}

In cases where $C^{4}$ effects are a small correction, we may solve the dispersion relation (3.14) iteratively. That is, first solve the $\lambda=\infty$ equation $Q^{I} Q_{I}=0$ for $q_{5}$, and then plug that solution into the correction term and solve

$$
Q^{I} Q_{I}=\left.\frac{1}{4} \zeta(3) \alpha^{\prime 3} Q^{H} Q^{K} Q_{P} Q_{Q} C_{H}^{R S P} C^{Q} R S\right|_{\text {null } Q_{I}}
$$

for $q_{5}$. Explicitly evaluating the $\mathrm{AdS}_{5}$-Schwarzschild Weyl tensor, ${ }^{21}$ one finds

$$
\left.Q^{H} Q^{K} Q_{P} Q_{Q} C_{H}^{R S P} C^{Q}{ }_{R S K}\right|_{\text {null } Q_{I}}=24 \frac{z^{12}|\boldsymbol{q}|^{4}}{\left(z_{\mathrm{h}} \mathrm{R}\right)^{8}} .
$$

Eq. (3.19), and the arguments leading up to it, give the leading high-energy terms of the $C^{4}$-corrected dispersion relation. Readers wishing to see the full $C^{4}$-corrected equation of motion for comparison, without any high-energy approximation, may find it in Appendix A.

Eq. (3.19) is already more detailed than we need for our purposes - the specifics do not matter for our qualitative conclusions, and it is easy to understand where the parametric dependence of (3.19b) comes from without doing any explicit calculation, at least for the case $z \ll z_{\mathrm{h}}$ that will be of interest for studying $z \sim z_{\star}$. Giving a generic argument will help us to later discuss yet-higher derivative corrections, where exact expressions for the corrections are not generally known.

Schematically, the left-hand side of (3.19b) has the form

$$
g^{\bullet \bullet} g^{\bullet \bullet} g \bullet g^{\bullet \bullet} g \bullet \bullet g^{\bullet \bullet} Q \bullet Q \bullet Q \bullet Q \bullet C \bullet \bullet \bullet C \bullet \bullet \bullet,
$$

where we will use bullets $(\bullet)$ to denote 5 -dimensional indices without focusing on the details of how they are contracted. (i) The four powers of $Q$ • produce four powers of $E$ (as long

$\left.\overline{21\left(C_{0101}\right.}, C_{1212}, C_{0505}, C_{1515}\right)=\left(f, 1,-3,-f^{-1}\right) \times \mathrm{R}^{2} / z_{\mathrm{h}}^{4}$, with all other components determined by symmetry. 
as they are not contracted with each other). (ii) The six powers of the inverse metric give six powers of $z^{2} / \mathrm{R}^{2}$ for $z \ll z_{\mathrm{h}}$, for a total of $z^{12} / \mathrm{R}^{12}$. (iii) Finally, consider $C_{\text {.... for small }}$ $z$. First think about pure $\mathrm{AdS}_{5}$ space instead of $\mathrm{AdS}_{5}$-Schwarzschild. The Riemann tensor has size

$$
R_{J K L}^{I} \sim \frac{1}{z^{2}}
$$

by dimensional analysis (remembering that the overall normalization of the metric cancels in $\left.R_{b c d}^{a}\right)$. Lowering the upper index gives

$$
R_{I J K L} \sim \frac{\mathrm{R}^{2}}{z^{4}}
$$

Because pure AdS is a maximally symmetric space, the corresponding Weyl tensor (which is the traceless part of Riemann) vanishes. When we go from $\mathrm{AdS}_{5}$ to $\mathrm{AdS}_{5}-\mathrm{Sch}$ warzschild, the only change to the metric is to replace $f=1$ by $f=1-\left(z / z_{\mathrm{h}}\right)^{4}$, which introduces corrections whose relative size at small $z$ is $\left(z / z_{\mathrm{h}}\right)^{4}$. So (3.22) becomes

$$
R_{I J K L} \sim \frac{\mathrm{R}^{2}}{z^{4}}\left[1+O\left(\frac{z^{4}}{z_{\mathrm{h}}^{4}}\right)\right] .
$$

When we construct the Weyl tensor $C_{\alpha \beta \gamma \delta}$, we know that the first term (the AdS one) will cancel and vanish. So, in $\mathrm{AdS}_{5}$-Schwarzschild,

$$
C_{I J K L} \sim \frac{\mathrm{R}^{2}}{z^{4}} \times O\left(\frac{z^{4}}{z_{\mathrm{h}}^{4}}\right) \sim \frac{\mathrm{R}^{2}}{z_{\mathrm{h}}^{4}} .
$$

Now multiplying our considerations of (i) through (iii) above gives

$$
g^{\bullet \bullet} g^{\bullet \bullet} g^{\bullet \bullet} g^{\bullet \bullet} g^{\bullet \bullet} g \bullet Q \cdot Q \cdot Q \bullet Q \bullet C \bullet \bullet \bullet \cdot \bullet \bullet \sim\left(\frac{z^{2}}{\mathrm{R}^{2}}\right)^{6} \times E^{4} \times\left(\frac{\mathrm{R}^{2}}{z_{\mathrm{h}}^{4}}\right)^{2} \sim \frac{z^{12} E^{4}}{\left(z_{\mathrm{h}} \mathrm{R}\right)^{8}}
$$

for small $z$, consistent with the exact result (3.19b).

Now solving the 5-dimensional dispersion relation (3.19) for $q_{5}\left(q_{\mu}, x^{5}\right)$ gives

$$
q_{5} \simeq \sqrt{g_{55}\left(-q_{\mu} g^{\mu \nu} q_{\nu}+\frac{\varepsilon z^{12}|\boldsymbol{q}|^{4}}{z_{\mathrm{h}}^{8} \mathrm{R}^{2}}\right)}
$$

where

$$
\varepsilon \equiv \frac{24}{\mathrm{R}^{6}} \times \frac{1}{4} \zeta(3) \alpha^{\prime 3}=\frac{6 \zeta(3)}{\lambda^{3 / 2}}
$$

is small.

At this point, we could measure the parametric importance of the $C^{4}$ correction simply by comparing the relative sizes of the $\varepsilon z^{12}|\boldsymbol{q}|^{4} / z_{\mathrm{h}}^{8} \mathrm{R}^{2}$ and $-q_{\mu} g^{\mu \nu} q_{\nu}$ terms in (3.26) at $z \sim z_{\star}$. But, for the sake of being slightly more explicit, let's first use (3.26) to get the stopping distance integral (3.18):

$$
\ell_{\text {stop }} \simeq \int_{0}^{z_{\mathrm{h}}} d z \frac{|\boldsymbol{q}|\left[1-\frac{2 \varepsilon z^{10}}{z_{\mathrm{h}}^{8}}|\boldsymbol{q}|^{2}\right]}{\sqrt{-q^{2}+\frac{z^{4}}{z_{\mathrm{h}}^{4}}|\boldsymbol{q}|^{2}+\frac{\varepsilon z^{10}}{z_{\mathrm{h}}^{8}}|\boldsymbol{q}|^{4 f}}}
$$


where $q^{2} \equiv q_{\mu} \eta^{\mu \nu} q_{\nu}$ denotes the 4 -momentum virtuality. The $\lambda=\infty$ result (2.10) corresponds to $\varepsilon=0$. There are various features of the integrand in (3.28) that need to be discussed, but first let's look at the relative size of the $C^{4}$ correction at $z_{\star}(2.12)$. Under the square root in the denominator, the $-q^{2}$ and $z^{4}|\boldsymbol{q}|^{2} / z_{\mathrm{h}}^{4}$ terms are the same size at $z \sim z_{\star}$ — that's how $z_{\star}$ was determined in the first place. Since $z_{\star} \ll z_{\mathrm{h}}$, we have $f \simeq 1$, and the relative size of the correction term is

$$
\operatorname{Importance}\left(C^{4}\right) \sim \frac{\frac{\varepsilon z_{\star}^{10}}{z_{\mathrm{h}}^{8}} E^{4}}{-q^{2}} \sim \frac{\left(-q^{2}\right)^{3 / 2}}{\lambda^{3 / 2} E T^{2}} .
$$

Using (1.1) and (2.13), this may be rewritten as

$$
\operatorname{Importance}\left(C^{4}\right) \sim\left(\frac{\lambda^{-1 / 4} \ell_{\text {max }}}{\ell_{\text {stop }}}\right)^{6} \sim \lambda^{-1 / 2}\left(\frac{\lambda^{-1 / 6} \ell_{\text {max }}}{\ell_{\text {stop }}}\right)^{6},
$$

which gives the $R^{4}$ (red) line in fig. 1 ,

The numerator correction in (3.28) is less important at $z \sim z_{\star}$. The relative size of its correction to the $\lambda=\infty$ integrand is

$$
\frac{\frac{\varepsilon z^{10}}{z_{\mathrm{h}}^{8}} E^{2}}{1}
$$

which is smaller than (3.29a) at $z \sim z_{\star}$ by a factor of $-q^{2} / E^{2} \ll 1$. One seemingly disturbing feature of the numerator correction is its sign for large enough $z$. For

$$
z \gg\left(\frac{\lambda^{3 / 4} T}{E}\right)^{1 / 5} z_{\mathrm{h}}
$$

(which is much larger than $z_{\star}$ ) the integrand is large and negative. If the integral (3.28) is blindly integrated up to $z_{\mathrm{h}}$ as written, one would find a rather extreme case of fig. 4k. However, we show in sections [VI and Appendix [B that the expansion in higher-derivative corrections breaks down well before one reaches $z$ 's as large as (3.31). And so the situation, for stopping distances in the safe region $\ell_{\text {stop }} \gg \lambda^{-1 / 6} \ell_{\max }$ of fig. 1, is that of fig. 4a. Following our assumptions from section IC, we therefore stick to (3.29) as the measure of the importance of $C^{4}$ corrections.

As far as $C^{4}$ corrections are concerned, our main results in this paper (fig. 1) only require the parametric information (3.29) on the importance of the $C^{4}$ correction at $z \sim z_{\star}$. One

might be tempted to attempt to extract an exact size for the leading correction from the explicit integral (3.28). We show in Appendix B why this will fail. Eq. (3.29) is adequate for determining whether or not the correction will be small, given our assumptions in section IIC. But the appendix shows that, even when the correction is small, the integrand cannot be trusted for the range of $z$ values required to use (3.29) for a precise calculation of the correction.

\section{THE $D^{2 n} R^{4}$ CORRECTIONS}

In this section, we'll investigate our first sequence of higher and higher derivative corrections to the 10-dimensional supergravity dual, by looking at $R^{4}$ terms with higher and higher 
powers of covariant derivatives. (We'll save adding extra powers of the Riemann curvature for the next section.) The parametric size of such corrections can be studied in complete generality, without focusing on precise formulas for the terms $D^{2 n} R^{4}$. However, for the sake of being concrete and making contact with standard string theory results, we'll first pause to briefly review what's known about these operators in the case of duality with Type II string theory.

\section{A. Review: 4-point string amplitude}

Just like the $R^{4}$ interaction in supergravity arises from the low-energy limit of gravitongraviton scattering in string theory, the $D^{2 n} R^{4}$ operators arise by looking more generally at the energy/momentum dependence of that scattering. At tree level (appropriate for $N_{\mathrm{c}}=\infty$ ), the energy dependence of the amplitude is captured by an overall factor

$$
T(s, t, u)=-\frac{\Gamma\left(-\alpha^{\prime} s / 4\right) \Gamma\left(-\alpha^{\prime} t / 4\right) \Gamma\left(-\alpha^{\prime} u / 4\right)}{\Gamma\left(1+\alpha^{\prime} s / 4\right) \Gamma\left(1+\alpha^{\prime} t / 4\right) \Gamma\left(1+\alpha^{\prime} u / 4\right)},
$$

where $s, t$, and $u$ are the Mandelstam variables (in 10 dimensions). This result is an "onshell" result, which means it is derived for string scattering in a flat-space background with the external momenta on-shell. That is, the result assumes $q_{a} q^{a}=0$ for each of the four 10-dimensional external momenta, which means $s+t+u=0$. Expanding $T(s, t, u)$ in powers of momenta gives [29]

$$
\begin{aligned}
T & =\frac{64}{\alpha^{\prime 3} s t u} \exp \left[\sum_{n=1}^{\infty} \frac{2 \zeta(2 n+1)}{2 n+1}\left(\frac{\alpha^{\prime}}{4}\right)^{2 n+1}\left(s^{2 n+1}+t^{2 n+1}+u^{2 n+1}\right)\right] \\
& =\frac{3}{\sigma_{3}}+2 \zeta(3)+\zeta(5) \sigma_{2}+\frac{2}{3} \zeta^{2}(3) \sigma_{3}+\frac{1}{2} \zeta(7)\left(\sigma_{2}\right)^{2}+\frac{2}{3} \zeta(3) \zeta(5) \sigma_{2} \sigma_{3}+\cdots,
\end{aligned}
$$

where

$$
\sigma_{k} \equiv\left(\frac{\alpha^{\prime}}{4}\right)^{k}\left(s^{k}+t^{k}+u^{k}\right)
$$

The first term in the expansion (4.2) corresponds to scattering that occurs through the interchange of an intermediate graviton, and this process is already accounted for by the usual Einstein-Hilbert piece $R$ of the low-energy supergravity action. The second term in (4.2), when generalized to curved space, gives the $R^{4}$ interaction previously discussed in section III. In order, the remaining terms give interactions that are schematically of the form $D^{4} R^{4}, D^{6} R^{4}$, etc.

In what follows, we will look at the parametric size of the effects of these interactions. Readers may wonder why we should think about a derivative expansion $D^{2 n} R^{4}$, and worry about where that expansion breaks down, when we already know that the expansion sums up to (4.1). There are two reasons. First, it will turn out that in those cases where the expansion is breaking down it will also be the case that the "on-shell" assumption $q^{a} q_{a}=0$ for the graviton momenta will also break down. So it is safest to not make any explicit assumptions about the detailed form of the $D^{2 n} R^{4}$ interactions. Secondly, analyzing the derivative expansion will provide a useful warm-up to more generally analyzing higherderivative corrections $D^{2 n} R^{m}$, which, as shown in fig. 1, are equally important when the derivative expansion breaks down for $D^{2 n} R^{4}$. As we will see, everything goes wrong at the 
same time, and so the explicit formula (4.1) for the 4-point amplitude does not seem useful then.

\section{B. Factors of $\alpha^{\prime} Q Q$}

In section III, we examined the effects of an $\alpha^{\prime 3} C^{4}$ interaction and found a 5-dimensional dispersion relation for the linearized scalar field $\phi$ with schematic form

$$
Q^{I} Q_{I}=\left.\alpha^{3} g^{\bullet \bullet} g \bullet g^{\bullet \bullet} g \bullet g^{\bullet \bullet} g \bullet Q \bullet Q \bullet Q \bullet Q \bullet C \bullet \bullet \bullet C \bullet \bullet \bullet\right|_{\text {null } Q_{I}} .
$$

This arose from terms in the Lagrangian quadratic in $\phi$, with 5-dimensional form

$$
\alpha^{\prime 3}(\nabla \nabla \phi)(\nabla \nabla \phi) C C
$$

coming from the 10-dimensional $\alpha^{\prime 3} C^{4}$.

Forget about string theory expressions and just think about what would happen if we went from $\alpha^{\prime 3} C^{4}$ to something of the form $\alpha^{\prime 4} D^{2} C^{4}$. (Note that the additional factor of $\alpha^{\prime}$ that accompanies the $D^{2}$ is simply a consequence of dimensional analysis.) Naively, we might think that the largest contribution arises from the case where both of the new derivatives have 5-dimensional indices and hit $\phi$ 's, modifying the right-hand-side of (4.4) to include an additional factor of

$$
\alpha^{\prime} g^{\bullet \bullet} Q_{\bullet} Q_{\bullet}
$$

Here the new indices might contract with the other indices in (4.4) or with each other. If we then note that $Q_{\mu}$ grows like $E$, we might at first guess that the parametric size of the additional factor (4.6) could be as large as

$$
\alpha^{\prime} \times \frac{z^{2}}{\mathrm{R}^{2}} \times E \times E,
$$

but this is an overestimate. If all six $Q$ 's in

$$
\alpha^{\prime 4} g^{\bullet \bullet} g^{\bullet \bullet} g^{\bullet \bullet} g^{\bullet \bullet} g^{\bullet \bullet} g^{\bullet \bullet} g \bullet Q \bullet Q \bullet Q \bullet Q \bullet Q \bullet Q \bullet C \bullet \bullet \bullet C \bullet \bullet \bullet
$$

are contracted with indices of the two Weyl tensor factors, the result must vanish because $C_{I J K L}$ is anti-symmetric in $I J$ and $K L$. As a result, two of the $Q$ 's must contract with each other, and so the cost of the factor (4.6) is

$$
\alpha^{\prime} Q^{I} Q_{I}
$$

instead of (4.7). In the $\lambda=\infty$ calculation, $Q^{I} Q_{I}=0$. In our calculation here, the effects discussed earlier arising from the $C^{4}$ correction change this to (3.19),

$$
Q^{I} Q_{I} \sim \alpha^{\prime 3} \frac{z^{12} E^{4}}{\left(z_{\mathrm{h}} \mathrm{R}\right)^{8}}
$$

So the size of the factor (4.6) at $z \sim z_{\star}$ is

$$
\left.\alpha^{\prime} Q^{I} Q_{I}\right|_{z \sim z_{\star}} \sim \alpha^{\prime 4} \frac{z_{\star}^{12} E^{4}}{\left(z_{\mathrm{h}} \mathrm{R}\right)^{8}} \sim \frac{\left(-q^{2}\right)^{3}}{\lambda^{2} E^{2} T^{4}} \sim\left(\frac{\lambda^{-1 / 6} \ell_{\max }}{\ell_{\text {stop }}}\right)^{12} .
$$


As we will discuss, (4.6) is not, in fact, the dominant contribution for large $\ell_{\text {stop }}$ simply because of the suppression from having to contract the $Q$ 's. But let's focus on this type of contribution for a moment longer. First, (4.11) tells us that this particular contribution from $\alpha^{4} D^{2} C^{4}$ becomes just as important as $\alpha^{3} C^{4}$ when $\ell_{\text {stop }} \sim \lambda^{-1 / 6} \ell_{\max }$, and so this is our first example of the breakdown of the expansion in higher-derivative corrections depicted in Fig. 1. If we add yet another factor of $\alpha^{\prime} D^{2}$ to go to $\alpha^{\prime 5} D^{4} C^{4}$, and consider just the contributions of the form (4.6) for that factor as well, then we will get another factor of (4.11), which will also not be suppressed at $\ell_{\text {stop }} \lesssim \lambda^{-1 / 6} \ell_{\max }$. Finally, note that all of the effects discussed so far are arising from $\alpha^{\prime} Q^{I} Q_{I}$ factors, which is just the dominant piece of 10-dimensional $\alpha^{\prime} q^{a} q_{a}$ factors. These are precisely the sort of factors that are left out of standard string theory "on-shell" results for higher-derivative corrections $D^{2 n} R^{4}$, but they become important for $\ell_{\text {stop }} \lesssim \lambda^{-1 / 6} \ell_{\max }$.

\section{The dominant factors}

If we add a factor of $\alpha^{\prime} D^{2}$ and neither derivative hits a $\phi$, then there will be no powers of $E$ to compensate the suppression from $\alpha^{\prime}$. The dominant terms come from the case where one derivative hits a $\phi$ and the other hits the background field:

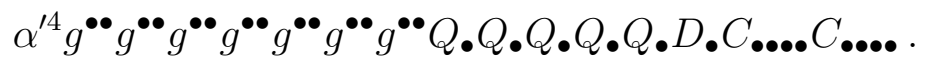

Since the background field depends only on $x^{5}$, it is natural to consider the case where the derivative $D_{\bullet}$ hitting the background Weyl tensor is a $D_{5}$. We will address this (relatively straightforward) case here. The contribution of terms involving other components $D_{\mu}$ of $D$. hitting the background is slightly more subtle and will be left to Appendix C.

Having the $D_{\bullet}$ which hits the background Weyl tensor be $D_{5}$ means (by 4 -dimensional parity invariance) that one of the $Q_{\bullet}$ 's must be $q_{5}$. Parametrically, a $D_{5}$ on the background Weyl tensor has size $z^{-1}$ for $z \ll z_{\mathrm{h}}$ (such as $z \sim z_{\star}$ ). So the factor of $\alpha^{\prime} D^{2}$ has cost

$$
\alpha^{\prime} g^{55} q_{5} D_{5}(\text { on bkgd }) \sim \alpha^{\prime} \times \frac{z^{2}}{\mathrm{R}^{2}} \times q_{5} \times z^{-1} .
$$

If you think of the factors in (4.5) as a 4-point amplitude with the two $\phi$ factors being legs 1 and 3 and the two $C$ factors being legs 2 and 4, then the cost shown above corresponds to (the curved background generalization of) an $\alpha^{\prime} s$ or $\alpha^{\prime} u$ factor in the string amplitude expansion (4.2).

For the size of $q_{5}$, we can just take the $\lambda=\infty$ result from $Q^{I} Q_{I} \simeq 0$,

$$
q_{5} \simeq \sqrt{g_{55}\left(-q_{\mu} g^{\mu \nu} q_{\nu}\right)} \simeq \sqrt{-q^{2}+\frac{z^{4}}{z_{\mathrm{h}}^{4}}|\boldsymbol{q}|^{2}}
$$

This is just (3.26) with $\varepsilon$ ignored. At $z \sim z_{\star}$, the two terms under the square root in (4.14) have comparable size, and so

$$
\left.q_{5}\right|_{z \sim z_{\star}} \sim \sqrt{-q^{2}}
$$

The cost (4.13) of $\alpha^{\prime} D^{2}$ is then

$$
\left.\alpha^{\prime} D^{2}\right|_{z \sim z_{\star}} \sim \frac{\alpha^{\prime} z_{\star} \sqrt{-q^{2}}}{R^{2}} \sim \frac{\left(-q^{2}\right)^{3 / 4}}{\lambda^{1 / 2} E^{1 / 2} T} \sim\left(\frac{\lambda^{-1 / 6} \ell_{\max }}{\ell_{\text {stop }}}\right)^{3} .
$$


This indeed dominates over (4.11) in the regime $\ell_{\text {stop }} \gg \lambda^{-1 / 6} \ell_{\max }$ where the expansion in higher-derivative corrections has not already broken down. Multiplying the importance (3.29) of $C^{4}$ by any number of factors (4.16) gives

$$
\text { Importance }\left(D^{2 n} C^{4}\right) \sim \lambda^{-1 / 2}\left(\frac{\lambda^{-1 / 6} \ell_{\max }}{\ell_{\text {stop }}}\right)^{3 n+6},
$$

which is shown by the $D^{2 n} R^{4}$ curves in fig. 1. Here and through most of this paper, we will be a little sloppy in distinguishing $R^{m}$ and $C^{m}$ in our discussion. We address that sloppiness in appendix $\mathrm{C} 4$ and show that it makes no difference for our qualitative conclusions.

\section{Beyond supergravity fields?}

Throughout this paper, in our treatment of the gravity dual, we focus on the dynamics of standard supergravity fields in $\mathrm{AdS}_{5}$-Schwarzschild. That is, we do not explicitly consider excited string states, which would correspond to a tower of extremely-massive fields in the supergravity theory, with masses of order $1 / \sqrt{\alpha^{\prime}}$. In our analysis, the effects of such excitations only appear indirectly through their effects on the effective interactions (such as $R^{4}$ ) between low-mass fields - that is, their only effects are as possible intermediate states in the string-scattering amplitudes which determine those effective interactions. One might wonder whether, for jets of sufficiently high energy $E$, it is possible to instead directly excite such high-mass states "on-shell" in the gravity picture. If so, we would need to explicitly include such fields in our analysis.

However, on-shell string excitations can be ignored precisely when the derivative expansion is under control, i.e. when $\ell_{\text {stop }} \gg \lambda^{-1 / 6} \ell_{\max }$ in fig. 1. To see this, consider, for example, the string-string scattering amplitude factor (4.1). This expression has poles when $\alpha^{\prime} s / 4$ (or similarly $\alpha^{\prime} t / 4$ or $\alpha^{\prime} u / 4$ ) is a non-negative integer, corresponding to an on-shell intermediate string state. To excite a massive string state therefore requires $s$ large enough that $\alpha^{\prime} s / 4 \geq 1$ (or similar for $\alpha^{\prime} t / 4$ or $\alpha^{\prime} u / 4$ ). But we've just seen in the previous sub-sections that $\alpha^{\prime} s$ and $\alpha^{\prime} t$ and $\alpha^{\prime} u$ are all parametrically small when $\ell_{\text {stop }} \gg \lambda^{-1 / 6} \ell_{\max }$. That is, the condition that the derivative expansion be under control is the same as the condition that the 5-dimensional Mandelstam variables are too small to create on-shell massive string excitations.

\section{HIGHER POWERS OF CURVATURE}

\section{A. $C^{5}$}

Now consider what happens if we include an additional factor of the Weyl tensor and look at an $\alpha^{4} C^{5}$ term in the 10-dimensional Lagrangian. There is, in fact, no independent $D^{2 n} C^{5}$ term with $n=0$ in Type II supergravity [20], but the $n=0$ case will make for a useful warm-up. The additional power of $\alpha^{\prime}$ that accompanies the additional power of $C$ is a consequence of dimensional analysis. In 5-dimensional language, the terms quadratic in the scalar field $\phi$ will have the form

$$
\alpha^{\prime 4}(\nabla \nabla \phi)(\nabla \nabla \phi) \bar{C} \bar{C} \bar{C}
$$


So all we've done by going from $\alpha^{\prime 3} C^{4}$ to $\alpha^{\prime 4} C^{5}$ is to modify the right-hand side of of the $C^{4}$ dispersion relation (4.4) to include an additional factor of

$$
\alpha^{\prime} g^{\bullet \bullet} g^{\bullet \bullet} C_{\bullet \bullet \bullet \bullet}
$$

[Since the Weyl tensor is traceless, the additional indices necessarily contract with the other indices in (4.4).] By the same parametric counting as in section IIIE, this new factor is (for $z \ll z_{\mathrm{h}}$ ) of order

$$
\alpha^{\prime} g^{\bullet \bullet} g^{\bullet \bullet} C_{\bullet \bullet \bullet} \sim \alpha^{\prime} \times\left(\frac{z^{2}}{\mathrm{R}^{2}}\right)^{2} \times \frac{\mathrm{R}^{2}}{z_{\mathrm{h}}^{4}} \sim \lambda^{-1 / 2} \frac{z^{4}}{z_{\mathrm{h}}^{4}} \ll 1
$$

There and no additional powers of energy to compensate the factor of $\lambda^{-1 / 2}$, and so a $C^{5}$ correction, if there were one, would always be small compared to a $C^{4}$ correction.

\section{B. $D^{2} C^{5}$}

Now add two more derivatives and instead consider $\alpha^{\prime 5} D^{2} C^{5}$. Naively, the dominant term is the one where both derivatives hit $\phi$ and give powers of $Q_{I}$ so that the net cost of adding factors $\alpha^{\prime 2} D^{2} C$ to the original $\alpha^{\prime 3} C^{4}$ is

$$
\alpha^{\prime 2} g^{\bullet \bullet} g^{\bullet \bullet} g \bullet Q_{\bullet} Q_{\bullet} C_{\bullet \bullet \bullet \bullet}
$$

Unlike what happened for $D^{2} C^{4}$ in section IVB, here the naive reasoning is correct because the two new factors of $Q$ need not contract with each other - they can instead contract with indices of the new factor of $C$ in the combination $Q^{I} Q^{K} C_{I J K L}$. The parametric size of (5.4) is therefore simply

$$
\alpha^{\prime 2} \times\left(\frac{z^{2}}{\mathrm{R}^{2}}\right)^{3} \times E^{2} \times \frac{\mathrm{R}^{2}}{z_{\mathrm{h}}^{4}} \sim \frac{z^{6} E^{2}}{\lambda z_{\mathrm{h}}^{4}} .
$$

At $z \sim z_{\star}$, this is

$$
\frac{z_{\star}^{6} E^{2}}{\lambda z_{\mathrm{h}}^{4}} \sim \frac{\left(-q^{2}\right)^{3 / 2}}{\lambda E T^{2}} \sim\left(\frac{\lambda^{-1 / 6} \ell_{\mathrm{max}}}{\ell_{\mathrm{stop}}}\right)^{6} .
$$

And so $D^{2} C^{5}$ becomes just as important as $C^{4}$ when $\ell_{\text {stop }} \sim \lambda^{-1 / 6} \ell_{\max }$, as in fig. 1.

\section{C. $D^{2 n} C^{m}$}

Every time we add another factor of $C$, we can also add a pair of large derivatives, just like above. For each new factor of $\alpha^{2} D^{2} C$ added, the cost is another factor of (5.6) . Multiplying $k$ such factors times the importance (3.29) of $C^{4}$ then gives

$$
\operatorname{Importance}\left(D^{2 k} C^{4+k}\right) \sim \lambda^{-1 / 2}\left(\frac{\lambda^{-1 / 6} \ell_{\max }}{\ell_{\text {stop }}}\right)^{6 k+6},
$$

which is depicted, for example, by the $D^{2} R^{5}$ and $D^{4} R^{6}$ lines in fig. 1. 
Starting from $D^{2 k} C^{4+k}$, we may then add further derivatives, but the parametric cost for additional derivatives will be the same as the discussion in section IVC, so that

$$
\operatorname{Importance}\left(D^{2 k+2 n} C^{4+k}\right) \sim \lambda^{-1 / 2}\left(\frac{\lambda^{-1 / 6} \ell_{\max }}{\ell_{\text {stop }}}\right)^{6 k+3 n+6}
$$

also depicted in fig. 1. The upshot is that, when considering higher-derivative terms $A$ in the supergravity Lagrangian, the subset with the dominant effect for a given engineering dimension $\operatorname{dim} A$ has importance

$$
\lambda^{-1 / 2}\left(\frac{\lambda^{-1 / 6} \ell_{\max }}{\ell_{\text {stop }}}\right)^{\frac{3}{2} \operatorname{dim} A-6}
$$

But we will need to finish our arguments, and consider other possible corrections, in order to verify this.

\section{Remaining details}

We've now seen the basic structure of corrections that give rise to fig. 1, but there are still a few details to clear up. So far, we have considered only powers of the Weyl curvature tensor. In appendix $[$, we show that it will not matter, qualitatively, if supergravity interactions instead involved the full Riemann tensor at some order in derivatives. Along the way, we also show that it is unimportant for the qualitative conclusions about the expansion that the first correction was $C^{4}$ rather than some lower power like $C^{2}$ and $C^{3}$. The appendix also addresses the effects of supergravity interactions involving the 5-form field strength $F$, which has a non-vanishing background value in $\left(\mathrm{AdS}_{5}-\mathrm{Schwarzschild}\right) \times S^{5}$.

\section{WHAT HAPPENS FOR $z \gg z_{\star}$ ?}

By making the reasonable assumptions that we outlined in section IIC, we have managed to analyze the question of when corrections become important by focusing on particle trajectories at $z \sim z_{\star}$. As $z$ increases beyond this scale, the forward progress of the trajectory slows to a stop as in fig. 4 a. We previously asserted that at some scale $z_{\text {bad }} \gg z_{\star}$ the expansion in higher-derivative corrections would eventually break down, as also depicted in fig. 4a, even if the expansion was well behaved at $z_{\star}$. Here we will take a moment to identify the size of $z_{\text {bad }}$.

Start by considering the cost of an $\alpha^{\prime} D^{2}$ factor, which we analyzed in section IVC for $z \sim z_{\star}$. At larger $z$, with $z_{\star} \lesssim z \ll z_{\mathrm{h}}$, (4.14) gives

$$
\left.q_{5}\right|_{z \gtrsim z_{\star}} \sim \frac{z^{2} E}{z_{\mathrm{h}}^{2}}
$$

Then the cost (4.13) of each $\alpha^{\prime} D^{2}$ factor is

$$
\left.\alpha^{\prime} D^{2}\right|_{z \gtrsim z_{\star}} \sim \frac{\alpha^{\prime} z q_{5}}{\mathrm{R}^{2}} \sim \frac{z^{3} E T^{2}}{\lambda^{1 / 2}}
$$


This cost is unsuppressed for $z \gtrsim z_{\text {bad }}$, where

$$
z_{\mathrm{bad}} \sim \frac{\lambda^{1 / 6}}{E^{1 / 3} T^{2 / 3}}
$$

The same constraint arises from the other important corrections that we analyzed. For instance, the cost (5.5) of adding an $\alpha^{\prime 2} D^{2} C$ factor was $z^{6} E^{2} / \lambda z_{\mathrm{h}}^{4}$, which also becomes unsuppressed at the same $z \gtrsim z_{\text {bad }}$.

Note that the requirement $\ell_{\text {stop }} \gg \lambda^{-1 / 6} \ell_{\max }$ for the expansion in corrections to be wellbehaved in fig. 1 is the same condition as requiring $z_{\text {bad }} \gg z_{\star}$.

\section{EXPONENTIAL TAILS}

So far our discussion of corrections has been parametric and therefore qualitative in nature. Our analysis has also relied on the geometric optics approximation and so only been valid for $\ell_{\text {stop }} \ll \ell_{\max }$, though we then parametrically extrapolated our results to $\ell_{\text {stop }} \sim \ell_{\max }$. We have seen that the expansion in higher-derivative corrections should be well-behaved at $\ell_{\text {stop }} \sim \ell_{\max }$, and the dominant correction is the $C^{4}$ term in the effective supergravity action. In this section, we will make an explicit calculation of the effect of this correction on a quantity related to the maximum stopping distance.

\section{A. The exponential tail scale $\ell_{\text {tail }}$}

We first need a crisp definition of the "maximum" stopping distance, or of something related to it. To that end, consider the sources that we have used so far in this paper. The source operator has been weighted with

$$
e^{i \bar{k} \cdot x} \Lambda_{L}(x)
$$

as in (2.4), where the envelope function $\Lambda_{L}(x)$ is localized within a distance $L$ in both space and time, and where

$$
\bar{k}=(E+\epsilon, 0,0, E-\epsilon) \quad \text { with } \quad \frac{1}{L} \ll \epsilon \ll E
$$

as in (2.7). The 4 -virtuality $-q^{2}$ of the source is $\simeq-\bar{k}^{2} \simeq 4 \epsilon E$. The support for the source function (7.1) in momentum space is depicted qualitatively in fig. 6a for the case (7.2). As we make $\epsilon$ (and so $-q^{2}$ ) smaller and smaller, the momenta move closer and closer to the line $q^{0}=q^{3}$ and the stopping distance becomes longer and longer. To include virtualities as small as possible, and so the longest possible stopping distances, we may instead just take a momentum distribution that straddles the $q^{0}=q^{3}$ line, as in fig. 6b. This just corresponds to (7.1) with

$$
\bar{k}=(E, 0,0, E)
$$

instead of (7.2). One should choose $L \ll \ell_{\max }$ so that the source is localized enough that the maximum stopping distance may in fact be determined from the system's response. As discussed in refs. [4, 5], the effect of a source like fig. 6b is to generate jets with different virtualities $-q^{2}$ on an event-by-event basis. The typical virtuality of points in the shaded 


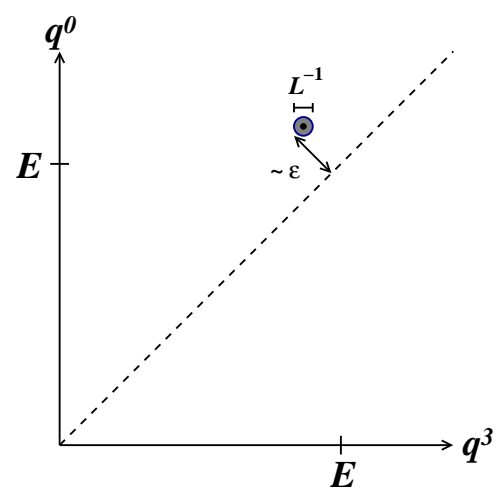

(a)

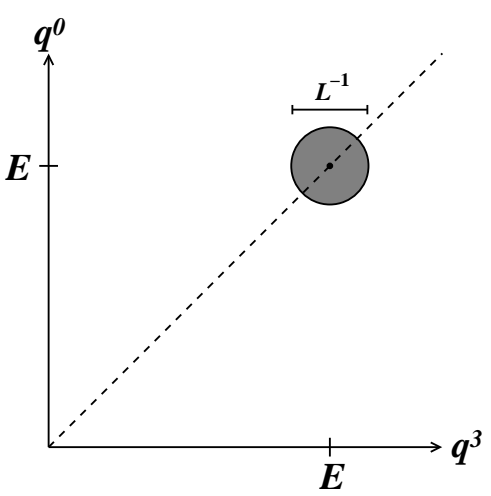

(b)

FIG. 6: Qualitative picture of momenta contributing to the source (2.4) used to generate jets (a) for the case $L^{-1} \ll \epsilon \ll E$ of (7.2), where one may use geometric optics, and (b) the case $\epsilon=0$, which has components with arbitrarily small $-q^{2}$ and which can generate jets with the largest stopping distances.

region of fig. $6 \mathrm{~b}$ is of order $E / L$, but some have much smaller virtualities. The result is a distribution of jet stopping distances from event to event. This distribution, as monitored by where the jet deposits thermalized charge into the system, has the qualitative form shown in fig. 7 [4]. The important point for our present purpose is that the distribution falls exponentially for distances large compared to the maximum stopping distance scale $\ell_{\max } \sim E^{1 / 3} T^{-4 / 3}$. By analyzing quasi-normal modes in the gravity dual, it is possible to calculate the scale that characterizes the rate of exponential fall-off. That is, it is possible to calculate what we will call " $\ell_{\text {tail }}$ " defined by (1.2),

$$
\operatorname{deposition}\left(x^{3}\right) \sim \text { prefactor } \times e^{-x^{3} / \ell_{\text {tail }}} \quad \text { for } \quad x^{3} \gg \ell_{\max } \text {. }
$$

The precise quantitative result for this tail scale $\ell_{\text {tail }}$ depends on the choice of source operator $O(x)$ used in the source (2.4) to generate the jet. As an example, for $\lambda=\infty$ and for $O(x)$ taken to be a transverse-polarized R-current, the result for the deposition tail is $[4]^{22}$

$$
\ell_{\text {tail }}\left(j^{\perp}\right) \simeq \frac{0.539 E^{1 / 3}}{(2 \pi T)^{4 / 3}} .
$$

The tail scale $\ell_{\text {tail }}$ is not a perfect stand-in for the maximum stopping distance $\ell_{\max }$. Consider the case of scalar source operators with large conformal dimension $\Delta \gg 1$ but $\Delta$ still parametrically small compared to powers of $E$ or $\lambda$. In ref. [5], it was shown that the corresponding $\ell_{\text {tail }}$ is $^{23}$

$$
\ell_{\text {tail }}(\Delta \gg 1) \simeq \frac{8 E^{1 / 3}}{3^{3 / 2} \Delta^{4 / 3}}
$$

but that the exponential fall-off characterized by (7.4) does not begin until a distance that is parametrically larger in $\Delta$ :

$$
\ell_{\max } \sim \frac{E^{1 / 3}}{\Delta^{1 / 3}}
$$

22 In the notation of ref. [4], the 0.539 in (7.5) above is $1 / 2 c_{1}$.

23 Specifically, see Appendix D of ref. [5] and eqs. (D2) and (D20) in particular. 


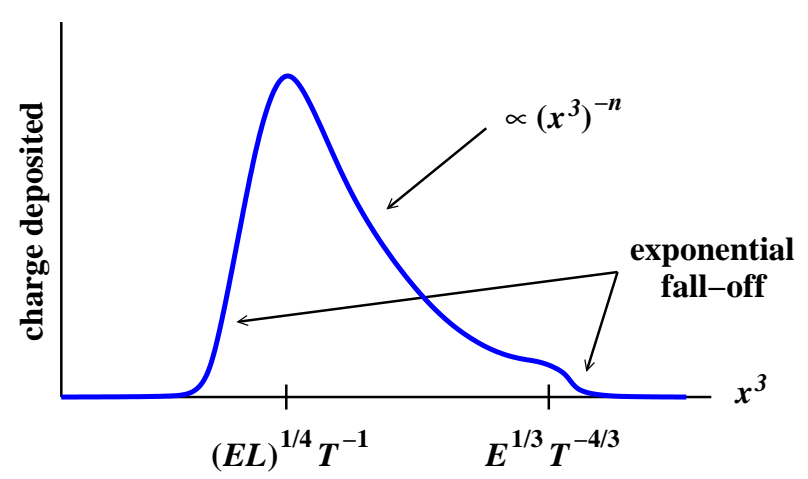

FIG. 7: The average deposition of charge as a function of $x^{3}$ for jets created by source momenta of the form of fig. 6 $\mathrm{b}$. The power $n$ of the algebraic fall-off for $(E L)^{1 / 4} T^{-1} \ll x^{3} \ll \ell_{\max }$ depends on the source operator [5].

This shows an example of how $\ell_{\text {tail }}$ can under-estimate the maximum stopping distance scale. Nonetheless, $\ell_{\text {tail }}$ is a specific, well-defined distance scale that is relatively easy to compute, and so we will use it as an example for a precise calculation of a $C^{4}$ correction related to jet stopping.

\section{B. $\ell_{\text {tail }}$ from quasi-normal modes}

As discussed in refs. [4, 5], the exponential tail in fig. 7 is determined by the pole of the high-energy boundary-to-bulk propagator that is closest to the real axis in 4-momentum space. ${ }^{24}$ Specifically, consider the light-cone components

$$
q^{ \pm} \equiv q^{3} \pm q^{0}, \quad q_{ \pm} \equiv \frac{1}{2} q^{\mp}=\frac{1}{2}\left(q^{3} \mp q^{0}\right)
$$

of 4-momentum $q$. At high energy $E$, the source given by (7.3) and fig. 6b has momenta with

$$
q_{-} \simeq E
$$

The more interesting component of the 4-momentum is therefore $q_{+}$. Consider the retarded boundary-to-bulk propagator as a function of $q_{+}$. A qualitative sketch of the pole structure in the complex $q_{+}$plane is shown in fig. $8{ }^{25}$ For large $x^{3}$, the exponential tail in fig. 7 is proportional to [4, 5]

$$
\left|e^{i q_{+}^{(0)} x^{+}}\right|^{2} \simeq e^{-4 x^{3} \operatorname{Im} q_{+}^{(0)}}
$$

where $q_{+}^{(0)}$ is the pole nearest the real axis, and deposition occurs near the light-cone $x^{0}=x^{3}$ in position space, so that $x^{+} \simeq 2 x^{3}$ [4]. The tail stopping scale $\ell_{\text {tail }}$ defined by (7.4) is

24 For related considerations of poles determining stopping distances, in the context of gluon beams created by synchrotron radiation, see section VI of ref. [6].

25 When comparing to refs. 44, 5], we have canceling differences in convention: (i) here we are discussing boundary-to-bulk propagators rather than bulk-to-boundary propagators, and (ii) our $q$ here is the 4 momentum associated with the bulk point rather than the boundary point. 


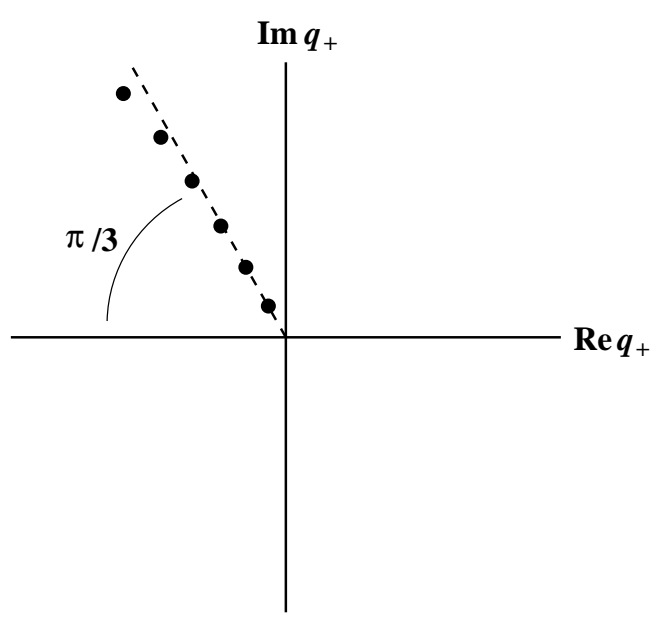

FIG. 8: A qualitative plot of the pole positions of the high-energy, retarded boundary-to-bulk propagator in the complex $q_{+}$plane. Note that the upper-half plane of $q_{+} \equiv \frac{1}{2}\left(q^{3}-q^{0}\right)$ corresponds to the lower-half plane of $q^{0}$.

therefore

$$
\ell_{\text {tail }}=\frac{1}{4 \operatorname{Im} q_{+}^{(0)}}
$$

We now need to find the pole position $q_{+}^{(0)}$. The locations of the poles correspond to quasi-normal modes, which are solutions that vanish at the boundary and have in-falling boundary conditions at the horizon, and which are associated with complex values of $q_{+}$.

\section{Finding quasi-normal mode solutions}

For $\lambda=\infty$, the equation of motion for a supergravity 5-dimensional scalar field in the $\mathrm{AdS}_{5}$-Schwarzschild background is $\left(-\nabla^{I} \nabla_{I}+m^{2}\right) \phi=0$, which is

$$
-\frac{1}{\sqrt{-g}} \partial_{5}\left(\sqrt{-g} g^{55} \partial_{5} \phi\right)+\left(q_{\mu} g^{\mu \nu} q_{\nu}+m^{2}\right) \phi=0 .
$$

The 5-dimensional mass term was unimportant to our previous analysis based on geometric optics for $\ell_{\text {stop }} \ll \ell_{\max }$, but it will be important for the determination of $\ell_{\text {tail }}$. At high energy, the dominant effect of $C^{4}$-corrections is to modify the equation of motion (7.12) as in $(3.19):{ }^{26}$

$$
-\frac{1}{\sqrt{-g}} \partial_{5}\left(\sqrt{-g} g^{55} \partial_{5} \phi\right)+\left(q_{\mu} g^{\mu \nu} q_{\nu}+m^{2}\right) \phi=\frac{\varepsilon z^{12}|\boldsymbol{q}|^{4}}{z_{\mathrm{h}}^{8} \mathrm{R}^{2}} \phi
$$

26 To obtain eq. (7.13), one may pick out the most important $C^{4}$ correction at high energy as outlined in section III. As a check, one may also start from a full equation of motion, such as (A4) for the case $k=0$, and pick out the dominant terms for (in units where $z_{\mathrm{h}}=1$ ) $|\boldsymbol{q}| \sim E \gg 1, z \sim E^{-1 / 3}$, and $-q^{2} \sim E^{2 / 3}$, which we will see are the relevant scales for determining the pole position. (This scaling is also described in ref. [30].) 
with $\varepsilon$ defined in (3.27). For the choice (2.18) of source operators in this paper, which are dual to the traceless part of $h_{\dot{a} \dot{b}}$ and have conformal dimension $\Delta=k+6$, the 5 -dimensional mass of the dual field in (7.12) and (7.13) is given by $[24]^{27}$

$$
(m \mathrm{R})^{2}=\Delta(\Delta-d)=(k+6)(k+2), \quad k=0,1,2, \cdots,
$$

where $d=4$ is the dimension of the boundary theory.

The derivation of the dominant $C^{4}$ correction also goes through identically for fluctuations $h_{12}$, dual to $T^{12}$. Recall that our convention is that the jet moves in the $x^{3}$ direction, and so by $h_{12}$ we mean fluctuations of the form $h_{12}\left(x^{0}, x^{3}\right)$ that do not depend on the transverse coordinates $\left(x^{1}, x^{2}\right)$. If one writes $h_{12}=z^{-2} \phi$, then the resulting equation of motion is identical to (7.13) in the high energy limit, but with ${ }^{28}$

$$
(m \mathrm{R})^{2}=0 \quad \text { for } h_{12} .
$$

On the field theory side of the duality, studying jet stopping with $T^{12}$ as our source operator can be thought of as studying the jet that would be created by the decay of a very highmomentum, slightly off-shell graviton in the 4-dimensional quark-gluon plasma.

Using the $\mathrm{AdS}_{5}$-Schwarzschild metric (2.1), the equation of motion (7.13) becomes

$$
-z^{3} \partial_{z}\left(z^{-3} f \partial_{z} \phi\right)+\left(\frac{q^{2}}{f}-\frac{z^{4}|\boldsymbol{q}|^{2}}{z_{\mathrm{h}}^{4} f}+\frac{(m \mathrm{R})^{2}}{z^{2}}-\frac{\varepsilon z^{10}|\boldsymbol{q}|^{4}}{z_{\mathrm{h}}^{8}}\right) \phi=0 .
$$

To find the high-energy quasi-normal modes, we will proceed as in ref. [5]..$^{29}$ As we will see, the solution for the quasi-normal mode will be dominated by its behavior at $z \sim$ $E^{-1 / 3} T^{-2 / 3} \ll z_{\mathrm{h}}$, and so we may approximate $f \simeq 1$ in (7.16). Then writing

$$
\phi=z^{3 / 2} \psi
$$

gives a Schrödinger-like equation

$$
-\frac{1}{2} \partial_{z}^{2} \psi+V(z) \psi=-\frac{1}{2} q^{2} \psi
$$

with potential

$$
V(z)=-\frac{z^{4}|\boldsymbol{q}|^{2}}{2 z_{\mathrm{h}}^{4}}+\frac{(m \mathrm{R})^{2}+\frac{15}{4}}{2 z^{2}}-\frac{\varepsilon z^{10}|\boldsymbol{q}|^{4}}{2 z_{\mathrm{h}}^{8}} .
$$

We may approximate $q^{2}=4 q_{-} q_{+} \simeq 4 E q_{+}$for our high-energy jets. Now change variables from $z$ to

$$
Z \equiv e^{-i \pi / 6} E^{1 / 3} z_{\mathrm{h}}^{-2 / 3} z
$$

so that (7.18) becomes

$$
-\frac{1}{2} \partial_{Z}^{2} \psi+\mathcal{V}(Z) \psi=\mathcal{E} \psi
$$

27 Our $k=0,1, \cdots$ differs by 2 from the $k=2,3, \cdots$ of eq. (2.47) of ref. [24], which in turn differs by 1 from the $k$ plotted for the $h_{(\alpha \beta)}$ curve in fig. 2 of ref. [24].

28 For comparison, the full $h_{12}$ equation of motion is given in (A7), and the dominant terms may be picked out as in footnote 26 .

29 Specifically appendix C.2 of ref. [5], but we will not make here the $\Delta \gg 1$ approximation used there. See ref. 30] for earlier work on high-energy quasi-normal modes which used an approximate method to solve the problem. Our $\lambda=\infty$ results agree with their approximate results parametrically but not in detail. 
with potential

$$
\begin{gathered}
\mathcal{V}(Z)=\frac{\mathcal{M}^{2}}{2 Z^{2}}+\frac{1}{2} Z^{4}-\frac{1}{2} \varepsilon Z^{10}, \\
\mathcal{M}^{2} \equiv(m \mathrm{R})^{2}+\frac{15}{4}
\end{gathered}
$$

and the "energy" $\mathcal{E}$ in the Schrödinger equation related to $q_{+}$by

$$
q_{+}=\frac{1}{2} e^{i 2 \pi / 3} z_{\mathrm{h}}^{-4 / 3} E^{-1 / 3} \mathcal{E}
$$

We want solutions to the original $\psi(z)$ equation (7.18) that, at large real $z$, only have waves moving away from the boundary (corresponding to having only in-falling waves at the horizon). Using the WKB approximation at large $z$, the large- $z$ behavior for $\varepsilon=0$, for example, would be

$$
\left.\psi(z)\right|_{\varepsilon=0} \sim e^{i z^{3}|\boldsymbol{q}| / 3 z_{\mathrm{h}}^{2}} .
$$

Such solutions correspond to solutions to the $\psi(Z)$ equation (7.21) that fall exponentially at large real values of $Z$, e.g.

$$
\left.\psi(Z)\right|_{\varepsilon=0} \sim e^{-Z^{3} / 3} \text {. }
$$

Quasi-normal modes are therefore solutions to (7.21) that (i) vanish at $Z=0$ and (ii) fall exponentially for large $Z{ }^{30}$ The allowed Schrödinger energies $\mathcal{E}$ in (7.21), which determine the quasi-normal mode values of complex $q_{+}$through (7.24), are therefore simply the bound state energies associated with the potential $\mathcal{V}(Z)$ of (17.22) in non-relativistic quantum mechanics.

Since we've argued in this paper that the $C^{4}$ correction term is small and under control for the physics associated with $\ell_{\text {stop }} \sim \ell_{\max }$, we should be able to treat the $\varepsilon Z^{10}$ term in the potential (7.22) as a perturbation. (We can double check that its effects are small at the end of the calculation.) The minimum value of $\mathcal{E}$, which determines the location of the $q_{+}$ pole closest to the real axis, can then be determined using ordinary perturbation theory as

$$
\mathcal{E}_{\min } \simeq \mathcal{E}_{0}-\frac{1}{2} \varepsilon\left\langle\psi_{0}\left|Z^{10}\right| \psi_{0}\right\rangle
$$

where $\mathcal{E}_{0}$ and $\psi_{0}(Z)$ are the ground state energy and wave function for a radial Schrödinger equation associated with a quartic potential $\frac{1}{2} Z^{4}$, with $\mathcal{M}$ playing the role of angular momentum. Combining (7.27) with (77.11) and (7.24) gives

$$
\ell_{\text {tail }}=\ell_{\text {tail }}^{\lambda=\infty}\left[1+\varepsilon \frac{\left\langle Z^{10}\right\rangle_{0}}{2 \mathcal{E}_{0}}+\cdots\right]
$$

with

$$
\ell_{\text {tail }}^{\lambda=\infty}=\frac{E^{1 / 3}}{\sqrt{3}(\pi T)^{4 / 3} \mathcal{E}_{0}} .
$$

30 One might worry about the negative sign in the $Z^{10}$ term in the potential (7.22), which seemingly implies that that $\mathcal{V}(Z)$ turns over and becomes negative for $Z \gg \varepsilon^{-1 / 6} \sim \lambda^{1 / 4}$. However, such extremely large values of $Z$ correspond to values of $|z|$ larger than the scale $z_{\text {bad }}$ of (6.3) where the expansion in higherderivative corrections breaks down. None of these issues will affect the perturbative evaluation of the shift in the pole position caused by the $\varepsilon Z^{10}$ term. 


\begin{tabular}{|c|c|c|c|c|c|c|}
\hline source operator & $\Delta$ & R charge rep. & SUGRA field & $(m \mathrm{R})^{2}$ & $\mathcal{E}_{0}$ & $\left\langle Z^{10}\right\rangle_{0}$ \\
\hline$T^{12}$ & 4 & trivial & $h_{12}$ & 0 & 4.4640 & 58.382 \\
$\operatorname{tr}(\lambda \lambda \bar{\lambda} \bar{\lambda})$ & 6 & $\underline{84}$ & traceless $h_{\dot{a} \dot{b}}$ & 12 & 8.5388 & 194.58 \\
\hline
\end{tabular}

TABLE I: Numerical results for $\mathcal{E}_{0}$ and $\left\langle Z^{10}\right\rangle_{0}$ for two examples of source operators. Also listed are the conformal dimension $\Delta$ and R-charge representation of the source operator, and the corresponding mass-squared of the supergravity (SUGRA) field.

Solving for $\psi_{0}(Z)$ numerically for various $m$ 's of interest gives results for $\mathcal{E}_{0}$ and $\left\langle Z^{10}\right\rangle_{0}$ shown in table I. Using (3.27) for $\varepsilon$, the corresponding formulas for $\ell_{\text {tail }}$ are

$$
h_{12}: \quad \ell_{\text {tail }}=\frac{0.3259 E^{1 / 3}}{(2 \pi T)^{4 / 3}}\left[1+47.162 \lambda^{-3 / 2}+O\left(\lambda^{-5 / 2}\right)\right]
$$

for source operator $T^{12}$ and

$$
h_{\dot{a} \dot{b}}(k=0): \quad \ell_{\text {tail }}=\frac{0.1704 E^{1 / 3}}{(2 \pi T)^{4 / 3}}\left[1+82.174 \lambda^{-3 / 2}+O\left(\lambda^{-5 / 2}\right)\right]
$$

for the lowest-dimension source operator $\operatorname{tr}(\lambda \lambda \bar{\lambda} \bar{\lambda})$ associated with traceless $h_{\dot{a} \dot{b}}$. The first case (7.30a) is the result that was previewed in the introduction (1.3).

We note that in both cases, the results for $\ell_{\text {tail }}^{\lambda=\infty}$ are quantitatively fairly well approximated by the large- $\mathcal{M}$ expression derived in ref. [5]: ${ }^{31}$

$$
\mathcal{M} \text { large }: \quad \ell_{\text {stop }}^{\lambda=\infty} \simeq \frac{E^{1 / 3}}{\sqrt{3}(2 \pi T)^{4 / 3}}\left[\frac{3}{8} \mathcal{M}^{4 / 3}+\sqrt{\frac{3}{8}} \mathcal{M}^{1 / 3}\right]^{-1} .
$$

However, we find that using the same approximation to calculate the $\lambda^{-3 / 2}$ correction term is a very poor approximation, basically because the large power in $\left\langle Z^{10}\right\rangle_{0}$ is extremely sensitive to the precise dependence of the wave function on $Z$.

\section{CONCLUSION}

At the beginning of this paper, we showed in fig. 1 the pattern of the parametric sizes of effects of higher-derivative supergravity corrections on the stopping distance. To demonstrate this pattern, we looked case by case at the different ways that higher-derivative terms might appear, and at various possible ways the indices in those terms might be contracted. We are suspicious that there must be a quicker, more elegant way to approach the power counting that would not require descending to that level of detail. It would be nice to figure out what the more elegant argument is, if there is one.

Also, our explicit results (7.28) and (7.30) for the leading correction to $\ell_{\text {tail }}$ fail to shed light on a mystery concerning the $\lambda$-dependence of stopping distances: How does the $E^{1 / 3}$

31 Specifically, this comes from the $n=0$ case of eq. (D19) of ref. [5], which gives what we call $2^{-4 / 3} \mathcal{E}_{0}$ in the present paper. Then use (7.29) in the present paper. The factor of $2^{-4 / 3}$ arises because the definitions of $Z$ in this paper and ref. [5] (and so the precise normalization of the quartic potential and the Schrödinger "energy") differ by a factor of $z_{\mathrm{h}}^{-2 / 3}$, which is a factor of $2^{2 / 3}$ in the units $2 \pi T=1$ used in ref. [5]. 
scaling of the maximum stopping distance at $\lambda=\infty$ transition to $E^{1 / 2}$ at small $\lambda$ ? One might naively guess the scaling to be of the form ${ }^{32}$

$$
\ell_{\max } \propto E^{f(\lambda)}
$$

for some function $f(\lambda)$ with $f(0)=\frac{1}{2}$ and $f(\infty)=\frac{1}{3}$. One might further hope that $f(\lambda)$ has relatively simple expansions around $\lambda=0$ and $\lambda=\infty$. For example, perhaps

$$
f(\lambda)=\frac{1}{2}+\# \lambda+\# \lambda^{2}+\cdots
$$

and

$$
f(\lambda)=\frac{1}{3}+\# \lambda^{-3 / 2}+\# \lambda^{-5 / 2}+\cdots,
$$

where the \# signs represent numerical coefficients. The details of the expansion don't matter - one can imagine there could be different powers of $\lambda$ than shown above, or factors of $\ln \lambda$ in the expansion, and so forth. But take (8.3) as an example. Then, expanding (8.1) around $\lambda=\infty$,

$$
\ell_{\max } \propto E^{1 / 3}\left[1+\# \lambda^{-3 / 2} \ln E+O\left(\lambda^{-5 / 2}\right)\right] .
$$

We might therefore expect if we compute something that is related to the maximum stopping distance, like $\ell_{\text {tail }}$, the corrections in powers of $1 / \lambda$ should also come with powers of $\ln E$, as above. But there is no sign of a $\ln E$ factor in (17.30).

Perhaps the exponent $f(\infty)=\frac{1}{3}$ does not receive corrections until a yet-higher power in $\lambda$, but we are unsure how a $\ln E$ could arise in yet-higher-order calculations of the shift of the quasi-normal mode pole. Or perhaps the exponent does not have an expansion in powers of $1 / \lambda$ but instead behaves like $\frac{1}{3}+\# e^{-\# \lambda}$ for large $\lambda$. Or perhaps the tail scale $\ell_{\text {tail }}$ is a misleading stand-in for $\ell_{\max }$, as is known to happen in the case of $\Delta \gg 1[5]$. Whatever the resolution, given the absence of a $\ln E$ in our result for $\ell_{\text {tail }}$, the question of how $E^{1 / 3}$ begins to make its way towards $E^{1 / 2}$ (and vice versa) remains an open question.

\section{Acknowledgments}

We thank Edmond Iancu for discussions about jet stopping results at $\lambda=\infty$, and we thank Simon Caron-Huot for discussions about higher-order corrections at zero temperature. This work was supported, in part, by the U.S. Department of Energy under Grant No. DE-FG0297ER41027.

\section{Appendix A: Full $C^{4}$-corrected dispersion relations}

In this appendix, we will give examples of the linearized equation of motion of the metric, to order $O\left(\alpha^{\prime}\right)^{4}$. This includes the $\alpha^{\prime 3}$ correction of the background geometry, as well as the effect of the (Weyl $)^{4}$ term in the supergravity action.

In this paper, we have generally considered fluctuations $h_{\dot{a} \dot{b}}$ that live on the $S^{5}$. There are a variety of Kaluza-Klein states of this form, but here we will consider those with the

32 We ignore here logarithmic energy dependence in the prefactor of the exponential. The small- $\lambda$ scaling is really $(E / \ln E)^{1 / 2}$, which is equivalent to including a log-of-log energy dependence in the exponent $f$ : $(E / \ln E)^{1 / 2}=\exp \left[\frac{1}{2}-\frac{1}{2} \ln \ln E\right]$. 
lowest mass, which transform under the $(2,0,2)=\underline{84}$ representation of $\mathrm{SU}(4)$ [24]. But we will also give similar results for fluctuations $h_{12}$ of the $\mathrm{AdS}_{5}$-Schwarzschild metric. For simplicity, we set $z_{\mathrm{h}}=1$ and $\mathrm{R}=1$ throughout this appendix.

We start with $h_{\dot{a} \dot{b}}$. In $\mathrm{SO}(6)$ language, the Young tableau of the $\underline{84}$ has the form of a box, with two rows and two columns. The corresponding spherical harmonic on $S^{5}$ is given by

$$
\begin{aligned}
h_{\dot{a} \dot{b}}= & \phi^{\mathbf{A B C D}}(x)\left[V_{((\dot{a}}^{\mathbf{A B}} V_{\dot{b}))}^{\mathbf{C D}}\right. \\
& -\frac{1}{N-2}\left(\delta^{\mathbf{A C}} V_{((\dot{a}}^{\mathbf{E B}} V_{\dot{b}))}^{\mathbf{E D}}+\delta^{\mathbf{B D}} V_{((\dot{a}}^{\mathbf{A E}} V_{\dot{b}))}^{\mathbf{C E}}-\delta^{\mathbf{B C}} V_{((\dot{a}}^{\mathbf{E A}} V_{\dot{b}))}^{\mathbf{E D}}-\delta^{\mathbf{A D}} V_{((\dot{a}}^{\mathbf{E B}} V_{\dot{b}))}^{\mathbf{E C}}\right) \\
& \left.+\frac{1}{(N-1)(N-2)}\left(\delta^{\mathbf{A C}} \delta^{\mathbf{B D}}-\delta^{\mathbf{B C}} \delta^{\mathbf{A D}}\right) V_{((\dot{a}}^{\mathbf{E F}} V_{\dot{b}))}^{\mathbf{E F}}\right],
\end{aligned}
$$

where the double round bracket notation stands for symmetrization and removal of a trace, $x$ denotes the non-compact coordinates, and $N=6$ for $S O(6)$, which is the isometry group of $S^{5}$. We have also defined the embedding scalar functions

$$
\begin{aligned}
& Y^{\mathbf{A}} Y^{\mathbf{A}}=1, \quad \mathbf{A}=1,2, \ldots 6, \\
& Y^{\mathbf{1}}+i Y^{\mathbf{2}}=\sin \left(\theta_{1}\right) \cos \left(\theta_{2}\right) e^{i \phi_{1}}, \quad Y^{\mathbf{3}}+i Y^{\mathbf{4}}=\sin \left(\theta_{1}\right) \sin \left(\theta_{2}\right) e^{i \phi_{2}}, \quad Y^{\mathbf{5}}+i Y^{\mathbf{6}}=\cos \left(\theta_{1}\right) e^{i \phi_{3}},
\end{aligned}
$$

and written the Killing vectors in terms of them,

$$
V_{\dot{c}}^{\mathbf{A B}}=V_{\dot{c}}^{[\mathbf{A B}]}=Y^{[\mathbf{A}} \partial_{\dot{c}} Y^{\mathbf{B}]} .
$$

The reason for choosing such a fluctuation is twofold. Firstly, $h_{\dot{a} \dot{b}}$ originates in a traceless metric fluctuation on $S^{5}$, and it does not mix to quadratic order with fluctuations of other fields. Secondly, in case one is interested in setting up a conserved charge measurement as in [4], $h_{\dot{a} \dot{b}}$, with its nontrivial spherical harmonic on the sphere, can be used as source for a jet carrying R-charge.

The equation of motion of one of the $\underline{84}$ scalars is

$$
\begin{aligned}
0 & =\frac{f(z)}{2 z^{3}} \frac{d^{2} \phi(z)}{d z^{2}}-\frac{1}{2 z^{4}}\left(3+z^{4}\right) \frac{d \phi(z)}{d z}-\frac{1}{2 z^{3}} \phi(z)\left(\frac{12}{z^{2}}+\boldsymbol{q}^{2}-\frac{E^{2}}{f(z)}\right) \\
+ & {\left[\frac{57}{8} \frac{d^{4} \phi(z)}{d z^{4}} f^{2}(z) z^{7}-\frac{57}{4} \frac{d^{3} \phi(z)}{d z^{3}} f(z)\left(15 z^{4}-7\right) z^{6}\right.} \\
& +\frac{z}{16} \frac{d^{2} \phi(z)}{d z^{2}}\left(3\left(9063 z^{12}-9668 z^{8}+2021 z^{4}-200\right)+220 z^{6} f(z) \boldsymbol{q}^{2}+228 z^{6} E^{2}\right) \\
& +\frac{1}{16} \frac{d \phi(z)}{d z}\left(3\left(14079 z^{12}-11772 z^{8}+2125 z^{4}-200\right)-220 z^{6}\left(11 z^{4}-7\right) \boldsymbol{q}^{2}+1596 z^{6} E^{2}\right) \\
& -\frac{z \phi(z)}{16 f^{2}(z)}\left(-36\left(30 z^{8}-59 z^{4}+269\right) z^{2} f^{2}(z)+\left(2025 z^{4}-1799\right) z^{4} f^{2}(z) \boldsymbol{q}^{2}\right. \\
& -\left(1023 z^{12}-3504 z^{8}+3705 z^{4}+600\right) E^{2} \\
- & \left.\left.-220 z^{6} f(z) \boldsymbol{q}^{2} E^{2}-50 z^{6} f^{2}(z)\left(\boldsymbol{q}^{2}\right)^{2}-114 z^{6} E^{4}\right)\right],
\end{aligned}
$$


where $\gamma$ is as defined in [25]:

$$
\gamma=\frac{1}{8} \zeta(3) \alpha^{\prime 3}
$$

This equation may be solved perturbatively in $\gamma: \phi(z)=\phi_{0}+\gamma \phi_{1}+\cdots$. After substituting the solution of the zeroth order in $\gamma$ equation of motion, $\phi_{0}$, (A4) becomes

$$
\begin{aligned}
0 & =\frac{f(z)}{2 z^{3}} \frac{d^{2} \phi_{1}(z)}{d z^{2}}-\frac{1}{2 z^{4}}\left(3+z^{4}\right) \frac{d \phi_{1}(z)}{d z}-\frac{1}{2 z^{3}} \phi_{1}(z)\left(\frac{12}{z^{2}}+\boldsymbol{q}^{2}-\frac{E^{2}}{f(z)}\right) \\
& +\frac{f(z)}{4} \frac{d \phi_{0}(z)}{d z}\left(3\left(-1767 z^{8}+4326 z^{4}-200\right)+1120 z^{6} \boldsymbol{q}^{2}\right) \\
& +\frac{\phi_{0}(z)}{2 z}\left(9\left(15 z^{12}-1540 z^{8}+1596 z^{4}-100\right)\right. \\
& \left.-z^{2}\left(1899 z^{8}-2221 z^{4}+75\right) \boldsymbol{q}^{2}+6 z^{2}\left(101 z^{8}-131 z^{4}+25\right) \frac{E^{2}}{f(z)}+48 z^{8}\left(\boldsymbol{q}^{2}\right)^{2}\right) .
\end{aligned}
$$

Now turn to the linearized equation of motion for $h_{12}$. If one defines $\phi(x)=h_{12} z^{2}$, then similar considerations yield

$$
\begin{gathered}
0=\frac{2 f(z)}{z} \frac{d^{2} \phi(z)}{d z^{2}}-\frac{2}{z^{2}}\left(3+z^{4}\right) \frac{d \phi}{d z}-\frac{2}{z} \phi\left(\boldsymbol{q}^{2}-\frac{E^{2}}{f(z)}\right) \\
+\gamma\left[\frac{37}{2} \frac{d^{4} \phi(z)}{d z^{4}} f^{2}(z) z^{9}-37 \frac{d^{3} \phi(z)}{d z^{3}} f(z) z^{8}\left(15 z^{4}-7\right)\right. \\
+\frac{z^{3}}{4} \frac{d^{2} \phi(z)}{d^{2} z}\left(15445 z^{12}-15340 z^{8}+2863 z^{4}-600+236 f(z) z^{6} \boldsymbol{q}^{2}+148 z^{6} E^{2}\right) \\
+\frac{z^{2}}{4} \frac{d \phi(z)}{d z}\left(-1235 z^{12}+8460 z^{8}-1225 z^{4}-600-236 z^{6}\left(11 z^{4}-7\right) \boldsymbol{q}^{2}+1036 z^{6} E^{2}\right) \\
+\frac{z^{3} \phi(z)}{4}\left(-200 z^{2}\left(9 z^{8}-2 z^{4}-2\right)-z^{4}\left(465 z^{4}-719\right) \boldsymbol{q}^{2}\right. \\
+\left(-2665 z^{12}+3968 z^{8}-719 z^{4}+600\right) \frac{E^{2}}{f^{2}(z)} \\
\left.\left.+236 z^{6} \boldsymbol{q}^{2} \frac{E^{2}}{f(z)}+74 z^{6}\left(\boldsymbol{q}^{2}\right)^{2}+74 z^{6} \frac{E^{4}}{f^{2}(z)}\right)\right]
\end{gathered}
$$

(This equation extends that of ref. [25] to the case of non-zero $\boldsymbol{q}$. ${ }^{33}$ Solving for $\phi=$

$\overline{33}$ In particular, setting $\boldsymbol{q}=0$ in our eq. (A7) reduces it to eq. (3.25) of ref. [25], after fixing an obvious typo in the latter. 
$\phi_{0}+\gamma \phi_{1}+\ldots$ order-by-order in $\gamma$ yields the following equation of motion for $\phi_{1}$ :

$$
\begin{gathered}
0=\frac{2 f(z)}{z} \frac{d^{2} \phi_{1}(z)}{d z^{2}}-\frac{2}{z^{2}}\left(3+z^{4}\right) \frac{d \phi_{1}}{d z}-\frac{2}{z} \phi_{1}\left(\boldsymbol{q}^{2}-\frac{E^{2}}{f(z)}\right) \\
+\frac{d \phi_{0}(z)}{d z} f(z) z^{2}\left(3171 z^{8}+2306 z^{4}-600+960 z^{6} \boldsymbol{q}^{2}\right) \\
+\phi_{0}(z)\left(-50\left(9 z^{8}-2 z^{4}-2\right) z^{5}+12\left(89 z^{8}-119 z^{4}+25\right) \frac{E^{2}}{f(z)}\right. \\
\left.-2\left(851 z^{8}-789 z^{4}+75\right) z^{3} \boldsymbol{q}^{2}+96 z^{9}\left(\boldsymbol{q}^{2}\right)^{2}\right) .
\end{gathered}
$$

\section{Appendix B: Why (3.29) cannot precisely determine $\Delta \ell_{\text {stop }}$}

Consider the safe region $\ell_{\text {stop }} \gg \lambda^{-1 / 6} \ell_{\text {max }}$ of fig. 1, where the effects of higher-dimensional supergravity interactions should be suppressed. The $R^{4}$ corrections then dominate the corrections at $z \sim z_{\star}$. We might then be tempted to use the explicit form (3.2) of the $R^{4}$ correction, combined with the particle-based formula (3.18) for the stopping distance, to explicitly calculate the first correction to the $\lambda=\infty$ result (2.13) for the stopping distance. In this appendix, we will discuss why that does not work.

We will start with (3.28),

$$
\ell_{\text {stop }} \simeq \int_{0}^{z_{\mathrm{h}}} d z \frac{|\boldsymbol{q}|\left[1-\frac{2 \varepsilon z^{10}}{z_{\mathrm{h}}^{8}}|\boldsymbol{q}|^{2}\right]}{\sqrt{-q^{2}+\frac{z^{4}}{z_{\mathrm{h}}^{4}}|\boldsymbol{q}|^{2}+\frac{\varepsilon z^{10}}{z_{\mathrm{h}}^{8}}|\boldsymbol{q}|^{4 f}}}
$$

\section{Numerator correction}

We earlier promised a discussion of why the potentially sign-changing behavior of the numerator correction in the $R^{4}$-corrected formula $(\underline{\mathrm{B} 1})$ for the stopping distance could be ignored. The disturbing features of this correction arise in the $z$ range given by (3.31),

$$
z \gg z_{\text {disturbing }} \sim\left(\frac{\lambda^{3 / 4} T}{E}\right)^{1 / 5} z_{\mathrm{h}} .
$$

First of all, notice that this difficulty only arises at all if $z_{\text {disturbing }}<z_{\mathrm{h}}$, which requires

$$
E \gg \lambda^{3 / 4} T
$$

Now compare (B2) and (6.3):

$$
z_{\text {disturbing }} \sim\left(\frac{E}{\lambda^{1 / 8} T}\right)^{2 / 15} z_{\text {bad }} .
$$

The inequality (B3) then gives

$$
z_{\text {disturbing }} \gg z_{\text {bad }},
$$

and so the numerator correction cannot be believed in the range of $z$ for which it becomes disturbing. 


\section{Denominator correction}

Dropping the numerator correction from (B1) leaves

$$
\ell_{\text {stop }} \simeq \int_{0}^{z_{\mathrm{h}}} d z \frac{|\boldsymbol{q}|}{\sqrt{-q^{2}+\frac{z^{4}}{z_{\mathrm{h}}^{4}}|\boldsymbol{q}|^{2}+\frac{\varepsilon z^{10}}{z_{\mathrm{h}}^{8}}|\boldsymbol{q}|^{4} f}}
$$

For simplicity, in what follows we will just analyze the case $E \gg \lambda^{3 / 4} T$.

In the integrand, look at the expression under the square root in the denominator. The relative importance of the $\varepsilon z^{10}$ term grows with increasing $z$. At what $z$ scale does it start to dominate? We know that, when the $R^{4}$ corrections are small, the $\varepsilon z^{10}$ term is a small correction at $z \sim z_{\star}$. But what about at larger $z$ ? For $z \gg z_{\star}$, the $z^{4}$ term under the square root dominates over the $-q^{2}$ term, so we should compare the $\varepsilon z^{10}$ term to the $z^{4}$ term. These are the same size at a scale $z_{\star \star} \gg z_{\star}$ given by

$$
z_{\star \star} \sim \frac{z_{\mathrm{h}}^{2 / 3}}{\varepsilon^{1 / 6}|\boldsymbol{q}|^{1 / 3}} \sim \frac{\lambda^{1 / 4}}{E^{1 / 3} T^{2 / 3}} \sim \frac{\lambda^{1 / 4}}{l_{\max } T^{2}},
$$

assuming that $z_{\star \star} \ll z_{\mathrm{h}}$ so that $f \simeq 1$. But $z_{\star \star} \ll z_{\mathrm{h}}$ follows from (B7) and our consideration of $E \gg \lambda^{3 / 4} T$.

Now calculate the correction $\Delta \ell$ to the stopping distance by subtracting the $\lambda=\infty$ result (2.10) from (Bי),

$$
\Delta \ell \equiv \ell_{\text {stop }}-\ell_{\text {stop }}^{\lambda=\infty} \simeq \int_{0}^{z_{\mathrm{h}}} d z\left[\frac{|\boldsymbol{q}|}{\sqrt{-q^{2}+\frac{z^{4}}{z_{\mathrm{h}}^{4}}|\boldsymbol{q}|^{2}+\frac{\varepsilon z^{10}}{z_{\mathrm{h}}^{8}}|\boldsymbol{q}|^{4} f}}-\frac{|\boldsymbol{q}|}{\sqrt{-q^{2}+\frac{z^{4}}{z_{\mathrm{h}}^{4}}|\boldsymbol{q}|^{2}}}\right]
$$

This integral is dominated by $z \sim z_{\star \star \star}$. So, to explicitly calculate $\Delta \ell$ will require trusting the integrand at $z \sim z_{\star \star}$ given by (B77). Compare this to the $z$ scale (6.3) where the expansion in supergravity corrections breaks down:

$$
z_{\star \star} \sim \lambda^{1 / 12} z_{\text {bad }} \gg z_{\text {bad }}
$$

So we cannot trust $(\underline{\mathrm{B} 8})$ in the range of $z$ where we want to use it to get an explicit result for $\Delta \ell$.

Note that, in order to use a particle-based formula such as (B8), one should check that the geometric optics approximation is actually valid at $z \sim z_{\star \star}$. Arguments similar to those of ref. [5] show that, for the $\lambda=\infty$ calculation, the geometric optics approximation is okay

for $z_{\mathrm{WKB}} \ll z \ll z_{\text {wave }}$ where $z_{\mathrm{WKB}} \sim 1 / \sqrt{-q^{2}}$ and $z_{\text {wave }} \sim-q^{2} / E T^{2}$. But discussing the resulting constraints is moot since we already have other reasons not to believe (B8) at $z \sim z_{\star \star}$.

\section{Appendix C: Other higher-derivative terms}

\section{1. $\alpha^{\prime} Q^{\mu} D_{\mu}$ contributions to $\alpha^{\prime} D^{2}$}

In section IVC, we focused on the $\alpha^{\prime} Q^{5} D_{5}$ piece of $\alpha^{\prime} D^{2}$ when studying the importance of $D^{2 n} C^{4}$. We also recycled our conclusion from that analysis when later considering applying 
extra powers of derivatives to $D^{2 k} C^{4+k}$. The dominant terms involved $\alpha^{\prime} Q^{I} D_{I}$ where the $D_{I}$ hits a background Weyl tensor. We motivated focusing on $Q^{5} D_{5}$ by noting that the background Weyl tensor depends only of the $x^{5}$ coordinate. If the $D$ 's were ordinary derivatives instead of covariant derivatives, that would be the end of the story. However, the other components $D_{\mu}$ of the covariant derivative do not vanish when applied to the background Weyl tensor. In fact, they are parametrically of order $1 / z$, just like $D_{5}$. As a result, for example,

$$
\alpha^{\prime} Q^{3} D_{3}=\alpha^{\prime} Q_{3} g^{33} D_{3} \sim \alpha^{\prime} \times E \times \frac{z^{2}}{\mathrm{R}^{2}} \times \frac{1}{z} \sim \frac{E z}{\lambda^{1 / 2}}
$$

is actually parametrically larger than the derivative

$$
\alpha^{\prime} Q^{5} D_{5} \sim \frac{q_{5} z}{\lambda^{1 / 2}}
$$

considered in the main text (4.13).

So why doesn't this lead to much larger results for the importance of $D^{2 n} R^{4}$ and other operators than shown in fig. 11? Our answer requires thinking about how the indices of the background Weyl tensor $C_{J K L M}$ hit by $\alpha^{\prime} Q^{I} D_{I}$ contract with everything else.

Because $C_{I J K L}$ depends only on $x^{5}$, a non-zero value for $Q^{\mu} D_{\mu} C_{I J K L}$ arises only from the terms of $D$ involving the Christoffel symbols:

$$
Q^{\mu} D_{\mu} C_{I J K L}=-Q^{\mu} \Gamma_{I \mu}^{\bar{I}} C_{\bar{I} J K L}-Q^{\mu} \Gamma_{J \mu}^{\bar{J}} C_{I \bar{J} K L}-\cdots
$$

Now write

$$
\Gamma=\Gamma^{(\mathrm{AdS})}+\Delta \Gamma,
$$

where $\Gamma^{(\mathrm{AdS})}$ is the zero-temperature, purely AdS expression for the connection $\Gamma$. The difference between AdS and $\mathrm{AdS}_{5}$-Schwarzschild is the difference between taking $f=1$ and $f=1-\left(z / z_{\mathrm{h}}\right)^{4}$ in the metric (2.1). As a result, the $\Delta \Gamma$ piece of (ㄷ) is suppressed compared to the $\Gamma^{(\mathrm{AdS})}$ piece by order $\left(z / z_{\mathrm{h}}\right)^{4}$. For studying the dominant corrections at $z \sim z_{\star} \ll z_{\mathrm{h}}$, we should therefore focus on $\Gamma^{(\operatorname{AdS})}$. In particular,

$$
\alpha^{\prime} Q^{\mu} \Delta \Gamma_{I \mu}^{\bar{I}} \sim \alpha^{\prime} E \times \frac{z^{2}}{\mathrm{R}^{2}} \times \frac{1}{z}\left(\frac{z}{z_{\mathrm{h}}}\right)^{4} \sim \frac{E z^{5}}{\lambda^{1 / 2} z_{\mathrm{h}}^{4}}
$$

is always less important at $z \sim z_{\star}$ than the $\alpha^{\prime} Q^{5} D_{5}$ term (C2) that we considered in the main text.

So now focus on $\Gamma^{\mathrm{AdS}}$ :

$$
Q^{\mu} D_{\mu} C_{I J K L} \simeq-Q^{\mu}\left(\Gamma_{I \mu}^{\bar{I}}\right)^{(\mathrm{AdS})} C_{\bar{I} J K L}-Q^{\mu}\left(\Gamma_{J \mu}^{\bar{J}}\right)^{(\mathrm{AdS})} C_{I \bar{J} K L}-\cdots
$$

Because zero-temperature, purely AdS space has 4-dimensional Lorentz invariance, the $\mu$ index on $Q^{\mu}$ above must pass through to contract with something else. For example,

$$
\begin{aligned}
Q^{\mu}\left(\Gamma_{I \mu}^{\bar{I}}\right)^{(\mathrm{AdS})} & C_{\bar{I} J K L} \times(\text { other stuff })^{I J K L} \\
& \simeq \frac{1}{z} Q^{\mu} C_{\mu J K L} \times(\text { other stuff })^{5 J K L}-\frac{1}{z} C^{5}{ }_{J K L} Q_{\mu} \times(\text { other stuff })^{\mu J K L}
\end{aligned}
$$

and

$$
\begin{aligned}
Q^{\mu}\left(\Gamma_{J \mu}^{\bar{J}}\right)^{(\mathrm{AdS})} & C_{I \bar{J} K L} \times(\text { other stuff })^{I J K L} \\
& \simeq \frac{1}{z} Q^{\mu} C_{I \mu K L} \times(\text { other stuff })^{I 5 K L}-\frac{1}{z} C_{I K L}^{5} Q_{\mu} \times(\text { other stuff })^{I \mu K L}
\end{aligned}
$$


But now recall that our dominant terms already had every $C$ contracted with two $Q$ 's. So the "other stuff" above had the form

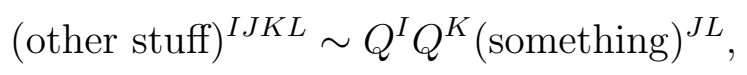

and these terms were dominant because both $Q_{I}$ and $Q_{K}$ were parametrically of order $E$ when contracted with the Weyl tensor $C_{I J K L}$. We are currently worried about the possibility that the $Q_{\mu}$ factor above is also of order $E$. Now look at the first term in (C7). The $Q_{\mu} Q_{I} Q_{K} \sim E^{3}$ is contracted in such a way that it instead gives $Q_{\mu} Q_{5} Q_{K} \sim q_{5} E^{2} \ll E^{3}$, which is not problematical. The second term in (C7) contracts two $Q$ 's together to give a factor of $Q_{\mu} \eta^{\mu \nu} Q_{\nu} \sim q^{2}$ instead of an $E^{2}$, and so it also is suppressed. Now look at the first term in (C8). There we have $Q^{\mu} Q^{I} Q^{K} C_{I \mu K L}$. Up to terms which are suppressed by $q_{5} \ll E$, this is the same as $Q^{J} Q^{I} Q^{K} C_{I J K L}$, which vanishes by the symmetry of the Weyl tensor. Finally, look at the second term of (C8), which involves

$$
\left.Q_{\mu} \text { (something }\right)^{\mu L} \text {. }
$$

For the dominant terms analyzed in the main text of this paper, the "something" is made up of factors of $Q$ and $Q Q C$. If (something) ${ }^{\mu L}$ gives a factor of $Q^{\mu}$, then two of our $Q$ 's that were supposed to be giving factors of $E$ will instead give a factor of $-q^{2} \ll E$. If (something) ${ }^{\mu L}$ gives a factor of $Q_{N} Q_{P} C^{N \mu P \bullet}$, then we'll get a suppression as before because of the symmetry of $C$.

The upshot is that the worrisome $\alpha^{\prime} Q^{\mu} D_{\mu}$ pieces of $\alpha^{\prime} D^{2}$ seem large in isolation but do not give large contributions when combined with the rest of the expression that they are a part of. The $Q^{\mu}$ has to contract with something else in the expression. The only other things there at leading order in energy are other factors of $Q$ and $Q Q C$. In either case, this contraction leads to additional suppressions so that $\alpha^{\prime} Q^{\mu} D_{\mu}$ does not give anything larger than what we already considered in the main text. The same reasoning applies to higher powers $\left(\alpha^{\prime} Q^{\mu} D_{\mu}\right)^{k}$.

The argument in this sub-section is particularly lacking in elegance. As mentioned in the conclusion, it would be nice to devise a more elegant argument.

\section{2. $C^{2}$ and $C^{3}$}

There are no $C^{2}$ and $C^{3}$ corrections to supergravity from tree-level Type II string theory, but it will make a later argument smoother if we show that such corrections would not change things, even were they present. An $\alpha^{\prime} C^{2}$ correction would generate a quadratic term for $\phi$ of the form

$$
\alpha^{\prime}(\nabla \nabla \phi)(\nabla \nabla \phi)
$$

which in turn would modify the dispersion relation to something of the form

$$
Q^{I} Q_{I}=\alpha^{\prime} g^{\bullet \bullet} g^{\bullet \bullet} Q_{\bullet} Q_{\bullet} Q_{\bullet} Q_{\bullet}+\cdots .
$$

That is,

$$
Q^{I} Q_{I}=\alpha^{\prime}\left(Q^{I} Q_{I}\right)^{2}+\cdots
$$

where the ellipses indicates other corrections like the effect of $C^{4}$. The $C^{2}$ correction displayed explicitly in (C13) will turn out to be relatively innocuous because the $Q$ 's had to 
be contracted together and $Q^{I} Q_{I}=0$ for $\lambda=\infty$. Let's compare the $\alpha^{\prime}\left(Q^{I} Q_{I}\right)^{2}$ term on the right-hand side of (C13) to the corresponding term from $C^{4}$ shown in (3.19). Using (4.10), the ratio of the two is

$$
\left.\frac{\alpha^{\prime}\left(Q^{I} Q_{I}\right)^{2}}{\alpha^{\prime 3} z^{12} E^{4} /\left(z_{\mathrm{h}} \mathrm{R}\right)^{8}}\right|_{z \sim z_{\star}} \sim \frac{\alpha^{\prime 4} z_{\star}^{12} E^{4}}{\left(z_{\mathrm{h}} \mathrm{R}\right)^{8}} \sim \frac{\left(-q^{2}\right)^{3}}{\lambda^{2} E^{2} T^{4}} \sim\left(\frac{\lambda^{-1 / 6} \ell_{\max }}{\ell_{\text {stop }}}\right)^{12} .
$$

That is, $C^{2}$ is much less important than $C^{4}$ for $\ell_{\text {stop }} \gg \lambda^{-1 / 6} \ell_{\max }$. If we multiply (C14) by the importance (3.29) of $C^{4}$, we can summarize as

$$
\operatorname{Importance}\left(C^{2}\right) \sim \lambda^{-1 / 2}\left(\frac{\lambda^{-1 / 6} \ell_{\max }}{\ell_{\text {stop }}}\right)^{18} .
$$

Now make the same analysis for the effect that an $\alpha^{2} C^{3}$ term in the supergravity action would have. The corresponding quadratic term for $\phi$ would be

$$
\alpha^{\prime 2}(\nabla \nabla \phi)(\nabla \nabla \phi) C
$$

Let's first follow the naive reasoning that the dominant term will be the one where the derivatives all give factors of $Q$. Then the dispersion relation takes the form

$$
Q^{I} Q_{I}=\alpha^{\prime 2} g^{\bullet \bullet} g^{\bullet \bullet} g^{\bullet \bullet} g^{\bullet \bullet} Q \bullet Q \bullet Q \bullet Q \bullet C \bullet \bullet \bullet+\cdots .
$$

Because of the symmetry of the Weyl tensor, two of the $Q$ 's must contract with each other. So the term shown on the right-hand side has size

$$
\alpha^{\prime 2} Q^{I} Q_{I} g^{\bullet \bullet} g^{\bullet \bullet} g^{\bullet \bullet} Q \bullet Q . C \bullet \bullet \bullet \sim \alpha^{\prime 2} Q^{I} Q_{I} \times\left(\frac{z^{2}}{\mathrm{R}^{2}}\right)^{3} \times E^{2} \times \frac{\mathrm{R}^{2}}{z_{\mathrm{h}}^{4}} .
$$

This term will also be relatively innocuous because of the contracted factor of $Q^{I} Q_{I}$. Using (4.10), its importance relative to the corresponding $C^{4}$ term shown in (3.19) is

$$
\left.\frac{\alpha^{\prime 2} Q^{I} Q_{I} z^{6} E^{2} /\left(z_{\mathrm{h}} \mathrm{R}\right)^{4}}{\alpha^{\prime 3} z^{12} E^{4} /\left(z_{\mathrm{h}} \mathrm{R}\right)^{8}}\right|_{z \sim z_{\star}} \sim \frac{\alpha^{\prime 2} z_{\star}^{6} E^{2}}{z_{\mathrm{h}}^{4} \mathrm{R}^{4}} \sim \frac{\left(-q^{2}\right)^{3 / 2}}{\lambda E T^{2}} \sim\left(\frac{\lambda^{-1 / 6} \ell_{\max }}{\ell_{\text {stop }}}\right)^{6} .
$$

So $C^{3}$, if there were such a correction, would also be much less important than $C^{4}$ for $\ell_{\text {stop }} \gg \lambda^{-1 / 6} \ell_{\max }$, and

$$
\operatorname{Importance}\left(C^{3}\right)_{\text {all } Q^{\prime} \mathrm{s}} \sim \lambda^{-1 / 2}\left(\frac{\lambda^{-1 / 6} \ell_{\max }}{\ell_{\text {stop }}}\right)^{12} .
$$

However, we learned in section IV C that factors $Q^{I} Q_{I}$ of contracted $Q$ 's are often beaten by contributions where one of the derivatives acts instead on the background curvature. In addition to (17), we also have contributions of the form

$$
\begin{aligned}
Q^{I} Q_{I} & =\alpha^{\prime 2} g^{\bullet \bullet} g^{\bullet \bullet} g^{\bullet \bullet} g^{\bullet \bullet} Q \bullet Q \cdot \nabla \cdot \nabla \bullet C \bullet \bullet \bullet+\cdots \\
& \sim \alpha^{\prime 2} \times\left(\frac{z^{2}}{\mathrm{R}^{2}}\right)^{4} \times E^{2} \times z^{-2} \times \frac{\mathrm{R}^{2}}{z_{\mathrm{h}}^{4}}+\cdots
\end{aligned}
$$


Compared to the corresponding $C^{4}$ contribution, the term shown on the right-hand side has relative importance

$$
\left.\frac{\alpha^{\prime 2} z^{6} E^{2} / z_{\mathrm{h}}^{4} \mathrm{R}^{6}}{\alpha^{\prime 3} z^{12} E^{4} /\left(z_{\mathrm{h}} \mathrm{R}\right)^{8}}\right|_{z \sim z_{\star}} \sim \frac{z_{\mathrm{h}}^{4} \mathrm{R}^{2}}{\alpha^{\prime} z_{\star}^{6} E^{2}} \sim \frac{\lambda^{1 / 2} E T^{2}}{\left(-q^{2}\right)^{3 / 2}} \sim \lambda^{-1 / 2}\left(\frac{\lambda^{-1 / 6} \ell_{\max }}{\ell_{\text {stop }}}\right)^{-6} .
$$

Multiplying by the importance (3.29) of $C^{4}$ gives

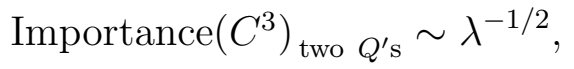

which is always small.

\section{Aside on Gauss-Bonnet gravity}

In the last sub-section, our evaluation of the importance of $C^{2}$ used the non-zero value (4.10) of $Q^{I} Q_{I}$ that is induced by the $C^{4}$ term. Though not relevant to our study of $\mathcal{N}=4 \mathrm{SYM}$, some readers may be curious what would happen if instead there were only $R^{2}$ corrections and all other higher-derivative corrections were absent. ${ }^{34}$ If we correspondingly throw away the "..." in the $C^{2}$ dispersion relation (C13) (and we'll see in the next subsection that it doesn't matter whether it is $C^{2}$ or $R^{2}$ ), then $Q^{I} Q_{I}=0$ remains a solution to the dispersion relation. So the geometric-optics based calculation of stopping distances can in this case be accomplished with the simple null geodesic formula (2.9) - the only difference is that one should use the $R^{2}$-corrected background metric instead of the unperturbed $\mathrm{AdS}_{5^{-}}$ Schwarzschild metric.

One might in particular consider the case of the $R^{2}$ correction to $\mathcal{N}=2 \quad D=5$ gauged supergravity (in contrast to the IIB string theory corrections to $\mathcal{N}=8 \quad D=5$ gauged supergravity relevant to this paper). Working perturbatively in the $R^{2}$ coupling these terms may be put into a Gauss-Bonnet form by a field redefinition of the metric. Let $\lambda_{\text {GB }}$ parametrize the coefficient of the Gauss-Bonnet $R^{2}$ term in the supergravity action. Then, assuming all yet-higher derivative terms in the Lagrangian are absent, the correction to the $\operatorname{AdS}_{5^{-}}$ Schwarzschild background is known to all orders in $\lambda_{\mathrm{GB}}$ [34, 35]. If one carries through the null geodesic calculation in this modified metric, it turns out that one finds the same $\ell_{\text {stop }} \propto\left(-q^{2} / E^{2}\right)^{1 / 4} / T$ result as in (2.13) , but the constant of proportionality is a function of $\lambda_{\mathrm{GB}} \cdot{ }^{35}$

\section{4. $\quad D^{2 n} R^{m}$ vs. $D^{2 n} C^{m}$}

In the main text, we considered only powers of the Weyl curvature tensor rather than the Riemann curvature tensor. Rather than spend time wondering whether one or the other arises from string interactions at all powers of curvature, we can dispense with the difference

34 For a discussion of the effects that Gauss-Bonnet gravity would have on other types of "jet" stopping calculations, such as drag forces on trailing classical strings or calculations of $\hat{q}$, see refs. 31-33].

35 Specifically, for $\lambda_{\mathrm{GB}}$ as defined in ref. [35] for $D=5$, we find (2.13) is modified by an overall factor of $[s(1+s) / 2]^{1 / 4}$ where $s \equiv \sqrt{1-4 \lambda_{\mathrm{GB}}}$. 
with a relatively simple argument. At first sight, using Riemann instead of Weyl might seem to make a dramatic difference in our previous power counting arguments because

$$
R_{I J K L} \sim \frac{\mathrm{R}^{2}}{z^{4}} \quad \gg \quad C_{I J K L} \sim \frac{\mathrm{R}^{2}}{z_{\mathrm{h}}^{4}}
$$

for the case $z \sim z_{\star} \ll z_{\mathrm{h}}$ of interest. But, even if there are such higher-derivative terms in the action with $R$ 's rather than $C$ 's, they do not cause a problem.

To see this, separate out the $z^{-4}$ piece of the Riemann tensor, which is just the zerotemperature Riemann tensor corresponding to AdS rather than $\mathrm{AdS}_{5}$-Schwarzschild. But the AdS Riemann tensor has a simple form in terms of the AdS metric:

$$
R_{\bullet \bullet \bullet}^{\mathrm{AdS}} \propto g_{\bullet \bullet}^{\mathrm{AdS}} g_{\bullet \bullet}^{\mathrm{AdS}} \text { (appropriately symmetrized) }
$$

The idea is to separate this piece from the Ads-Schwarzschild Riemann tensor, but to use the Ads-Schwarzschild metric instead of the AdS metric. So write

$$
R_{\bullet \bullet \bullet \bullet}=R_{\bullet \bullet \bullet \bullet}^{(0)}+(\Delta R)
$$

where

$$
R_{I J K L}^{(0)}=\frac{1}{\mathrm{R}^{2}}\left(g_{I L} g_{J K}-g_{I K} g_{J L}\right),
$$

and where, for $z \ll z_{\mathrm{h}}$,

$$
(\Delta R)_{\bullet \bullet \bullet \bullet} \equiv R_{\bullet \bullet \bullet \bullet}-R_{\bullet \bullet \bullet \bullet}^{(0)} \sim \frac{\mathrm{R}^{2}}{z_{\mathrm{h}}^{4}}
$$

is parametrically the same size as $C$ ! We never used the tracelessness of $C$ in our previous estimates, and so our earlier power counting of interactions with $C$ 's will also work for interactions with $\Delta R$ 's. As a result, the only thing we have to worry about are interactions that contain powers of $R^{(0)}$. But adding a factor of (C27) does not produce a new type of interaction - it just costs a power of $\alpha^{\prime}$. For example,

$$
\alpha^{\prime 4} C^{4} R^{(0)} \sim \frac{\alpha^{\prime 4}}{\mathrm{R}^{2}} C^{4} \sim \lambda^{-1 / 2} \alpha^{\prime 3} C^{4}
$$

This is small compared to the usual $\alpha^{\prime 3} C^{4}$ term. Similarly,

$$
\alpha^{\prime 4} C^{3}\left(R^{(0)}\right)^{2} \sim \frac{\alpha^{\prime 4}}{\mathrm{R}^{4}} C^{3} \sim \lambda^{-1} \alpha^{\prime 2} C^{3}
$$

is small compared to an $\alpha^{2} C^{3}$ term, which we saw in section C2 was small in turn. All terms involving $R^{(0)}$ will be small by powers of $\lambda^{-1 / 2}$ compared to corrections that we have previously analyzed.

\section{Corrections involving the 5-form $F$}

The $\left(\mathrm{AdS}_{5}\right.$-Schwarzschild $) \times S^{5}$ background involves a non-vanishing self-dual 5 -form field strength $F$. So we should check if we can get any important corrections from terms like $D^{2 n} R^{m} F^{2 k}$ or $D^{2 n+1} R^{m} F^{2 k+1}[36]$ in the supergravity Lagrangian - that is, by adding factors 
of $\alpha^{\prime} \bar{F}^{2}$ or $\alpha^{\prime} \bar{F} D$ to the dispersion relations we have considered previously. The background value is

$$
\bar{F}_{I J K L M}=\frac{1}{\mathrm{R}} \sqrt{-g^{(5)}} \epsilon_{I J K L M}
$$

in the $\mathrm{AdS}_{5}$-Schwarzschild space (and the dual of this on $S^{5}$ ), where $\epsilon_{I J K L M}$ is the 5component anti-symmetric symbol. Because of our choice back in section IID to choose a source operator that is dual to components of $h_{a b}$ which do not mix with $F$, the only relevance of $F$ in our problem is its background value $\bar{F}$. For simplicity, we will focus on the terms where the indices of $\bar{F}$ live in $\mathrm{AdS}_{5}$-Schwarzschild rather than $S^{5}$.

An even number of factors of $\bar{F}$ then means an even number of factors of 5-dimensional $\sqrt{-g} \epsilon$, and an even number of such factors may always be rewritten in terms of the metric tensor:

$$
-g^{(5)} \epsilon_{\bullet \bullet \bullet \bullet} \epsilon_{\bullet \bullet \bullet \bullet \bullet} \propto g_{\bullet \bullet} g_{\bullet \bullet} g_{\bullet \bullet} g_{\bullet \bullet} g_{\bullet \bullet} \text { (appropriately symmetrized) } .
$$

Because of this, the power counting involved in adding a factor of $\alpha^{\prime} F^{2}$ is similar to the previous discussion in section $\mathrm{C} 4$ of adding a factor of $\alpha^{\prime} R^{(0)}$, and so all such corrections are suppressed compared to the other corrections previously considered.

What about correction terms with an odd number of $F$ 's, such as $D^{2 n+1} R^{m} F^{2 k+1}$ ? The jet-stopping problem we have chosen to study is reflection invariant in the spatial dimensions $\left(x^{1}, x^{2}\right)$ transverse to the direction of the jet, e.g. $x^{1} \leftrightarrow x^{2}$. Specifically, the background $\mathrm{AdS}_{5}$-Schwarzschild metric has this invariance and, by choosing the source to be invariant as in (2.6), the 5-dimensional response $\phi\left(x^{\mu}, x^{5}\right)$ will be invariant as well. So the supergravity action will only be relevant for the case where $F$ takes on its background value and all the other fields are transverse-reflection invariant in $\left(x^{1}, x^{2}\right)$. But, with these restrictions, a term in the action with an odd number of $\epsilon_{I J K L M}$ 's from an odd number of $\bar{F}$ 's will contract to zero. And so such terms may be ignored for our problem.

[1] S. S. Gubser, D. R. Gulotta, S. S. Pufu and F. D. Rocha, "Gluon energy loss in the gauge-string duality," JHEP 0810, 052 (2008) [arXiv:0803.1470 [hep-th]].

[2] Y. Hatta, E. Iancu and A. H. Mueller, "Jet evolution in the $\mathcal{N}=4$ SYM plasma at strong coupling," JHEP 0805, 037 (2008) [arXiv:0803.2481 [hep-th]].

[3] P. M. Chesler, K. Jensen, A. Karch and L. G. Yaffe, "Light quark energy loss in strongly-coupled $\mathcal{N}=4$ supersymmetric Yang-Mills plasma," Phys. Rev. D 79, 125015 (2009) arXiv:0810.1985 [hep-th]].

[4] P. Arnold, D. Vaman, "Jet quenching in hot strongly coupled gauge theories revisited: 3-point correlators with gauge-gravity duality," JHEP 1010, 099 (2010) arXiv:1008.4023 [hep-th]].

[5] P. Arnold and D. Vaman, "Jet quenching in hot strongly coupled gauge theories simplified," JHEP 1104, 027 (2011) arXiv:1101.2689 [hep-th]].

[6] P. M. Chesler, Y. -Y. Ho and K. Rajagopal, "Shining a Gluon Beam Through Quark-Gluon Plasma," arXiv:1111.1691 [hep-th].

[7] S.-J. Sin and I. Zahed, "Holography of radiation and jet quenching," Phys. Lett. B 608, 265 (2005) hep-th/0407215.

[8] M. Spillane, A. Stoffers and I. Zahed, "Jet quenching in shock waves," JHEP 1202, 023 (2012) arXiv:1110.5069 [hep-th]]; A. Stoffers and I. Zahed, arXiv:1110.2943 [hep-th]. 
[9] C. P. Herzog, A. Karch, P. Kovtun, C. Kozcaz and L. G. Yaffe, "Energy loss of a heavy quark moving through N=4 supersymmetric Yang-Mills plasma," JHEP 0607, 013 (2006) hep-th/0605158.

[10] J. Casalderrey-Solana and D. Teaney, "Heavy quark diffusion in strongly coupled $\mathcal{N}=4$ YangMills," Phys. Rev. D 74, 085012 (2006) hep-ph/0605199].

[11] P. B. Arnold, S. Cantrell and W. Xiao, "Stopping distance for high energy jets in weaklycoupled quark-gluon plasmas," Phys. Rev. D 81, 045017 (2010) arXiv:0912.3862 [hep-ph]].

[12] R. Baier, Y. L. Dokshitzer, A. H. Mueller, S. Peigné and D. Schiff, "The Landau-PomeranchukMigdal effect in QED," Nucl. Phys. B 478, 577 (1996) arXiv:hep-ph/9604327); "Radiative energy loss of high energy quarks and gluons in a finite-volume quark-gluon plasma," Nucl. Phys. B 483, 291 (1997) arXiv:hep-ph/9607355; "Radiative energy loss and $p_{\perp}$-broadening of high energy partons in nuclei," Nucl. Phys. B 484, 265 (1997) arXiv:hep-ph/9608322.

[13] B. G. Zakharov, "Fully quantum treatment of the Landau-Pomeranchuk-Migdal effect in QED and QCD," JETP Lett. 63, 952 (1996) arXiv:hep-ph/9607440]; "Radiative energy loss of high energy quarks in finite-size nuclear matter and quark-gluon plasma," JETP Lett. 65, 615 (1997) arXiv:hep-ph/9704255.

[14] H. Liu, K. Rajagopal, U. A. Wiedemann, "Calculating the jet quenching parameter from AdS/CFT," Phys. Rev. Lett. 97, 182301 (2006) hep-ph/0605178].

[15] P. Arnold, D. Vaman, "Some new results for 'jet' stopping in AdS/CFT," arXiv:1106.1680, an abridged version appears in J. Phys. G 38, 124175 (2011).

[16] N. Armesto, J. D. Edelstein and J. Mas, "Jet quenching at finite "t Hooft coupling and chemical potential from AdS/CFT," JHEP 0609, 039 (2006) hep-ph/0606245.

[17] P. Arnold and D. Vaman, "4-point correlators in finite-temperature AdS/CFT: Jet quenching correlations," JHEP 1111, 033 (2011) arXiv:1109.0040 [hep-th]].

[18] E. Shuryak, H. -U. Yee and I. Zahed, "Self-force and synchrotron radiation in odd space-time dimensions," arXiv:1111.3894 [hep-th].

[19] P. M. Chesler, K. Jensen and A. Karch, "Jets in strongly-coupled $\mathcal{N}=4$ super Yang-Mills theory," Phys. Rev. D 79, 025021 (2009) [arXiv:0804.3110 [hep-th]].

[20] S. Stieberger, "Constraints on Tree-Level Higher Order Gravitational Couplings in Superstring Theory," Phys. Rev. Lett. 106, 111601 (2011) arXiv:0910.0180 [hep-th]].

[21] D. M. Richards, "The One-Loop Five-Graviton Amplitude and the Effective Action," JHEP 0810, 042 (2008) arXiv:0807.2421 [hep-th]].

[22] D. J. Gross and E. Witten, "Superstring Modifications of Einstein's Equations," Nucl. Phys. B 277, 1 (1986).

[23] E. D'Hoker and D. Z. Freedman, "Supersymmetric gauge theories and the AdS / CFT correspondence," hep-th/0201253.

[24] H. J. Kim, L. J. Romans and P. van Nieuwenhuizen, "Mass spectrum of chiral ten-dimensional $N=2$ Supergravity on $S^{5}$," Phys. Rev. D 32, 389 (1985).

[25] A. Buchel, J. T. Liu and A. O. Starinets, "Coupling constant dependence of the shear viscosity in $\mathcal{N}=4$ supersymmetric Yang-Mills theory," Nucl. Phys. B 707, 56 (2005) hep-th/0406264.

[26] M. T. Grisaru and D. Zanon, "Sigma Model Superstring Corrections to the Einstein-Hilbert Action," Phys. Lett. B 177, 347 (1986); M. D. Freeman, C. N. Pope, M. F. Sohnius and K. S. Stelle, "Higher Order Sigma Model Counterterms and the Effective Action for Superstrings," Phys. Lett. B 178, 199 (1986); Q-H. Park and D. Zanon, "More on $\sigma$-model $\beta$ functions and low-energy effective actions," Phys. Rev. D 35, 4038 (1987).

[27] O. Aharony, S. S. Gubser, J. M. Maldacena, H. Ooguri and Y. Oz, "Large N field theories, 
string theory and gravity," Phys. Rept. 323, 183 (2000) hep-th/9905111.

[28] R. Kallosh and A. Rajaraman, "Vacua of M theory and string theory," Phys. Rev. D 58, 125003 (1998) hep-th/9805041.

[29] M. B. Green and P. Vanhove, "Low-energy expansion of the one loop type II superstring amplitude," Phys. Rev. D 61, 104011 (2000) hep-th/9910056].

[30] G. Festuccia and H. Liu, "A Bohr-Sommerfeld quantization formula for quasinormal frequencies of AdS black holes," arXiv:0811.1033 [gr-qc].

[31] K. B. Fadafan, " $R^{2}$ curvature-squared corrections on drag force," JHEP 0812, 051 (2008) arXiv:0803.2777 [hep-th]].

[32] K. B. Fadafan, "Charge effect and finite 't Hooft coupling correction on drag force and Jet Quenching Parameter," Eur. Phys. J. C 68, 505 (2010) arXiv:0809.1336 [hep-th]].

[33] J. Noronha, M. Gyulassy and G. Torrieri, "Constraints on AdS/CFT Gravity Dual Models of Heavy Ion Collisions," Phys. Rev. C 82, 054903 (2010) arXiv:0906.4099 [hep-ph]].

[34] R.-G. Cai, "Gauss-Bonnet black holes in AdS spaces," Phys. Rev. D 65, 084014 (2002) hep-th/0109133.

[35] A. Buchel, J. Escobedo, R. C. Myers, M. F. Paulos, A. Sinha and M. Smolkin, "Holographic GB gravity in arbitrary dimensions," JHEP 1003, 111 (2010) [arXiv:0911.4257 [hep-th]].

[36] M. F. Paulos, "Higher derivative terms including the Ramond-Ramond five-form," JHEP 0810, 047 (2008) arXiv:0804.0763 [hep-th]]. 